\section{Análisis de la integración y dependencia de las políticas monetarias de la Unión Eurapea}

Ma Carmen González Velasco ${ }^{1}$

Universidad de León carmen.gvelasco@unileon.es

Roque Brinckmann

Universidad Federal de

Santa Catarina roque@cse.ufsc.br

\title{
Resumen
}

En ese artículo se efectúa un análisis de la integración y dependencia de las políticas monetarias de la Unión Europea y, en concreto, de las políticas monetarias de la Unión Económica y Monetaria y de la zona no euro para el periodo comprendido entre Enero de 1999 y Septiembre 2009. Se aplica la metodología de la cointegración de Engle y Granger (1987) y de Johansen (1988) para contrastar la hipótesis de la paridad de tipos de interés no cubierta y se llega a la conclusión de que ambas políticas están cointegradas porque mantienen una relación de equilibrio a largo plazo. También se deduce una dependencia de la política del Banco de Inglaterra de la política del Banco Central Europeo, lo que confirma la importancia y el liderazgo de la Unión Económica y Monetaria.

Palabras clave: Política monetaria; Tipos interbancarios; Mercado interbancario; Cointegración; Dependencia; Paridad descubierta de intereses.

\section{Abstract}

This study is to investigate the long-run relationship and dependence between the UME's monetary policy and non-euro zone's monetary policy for the period from January 4, 1999 to September 30, 2009. We use cointegration methodology to test the Uncovered Interest Parity Hypothesis and the results indicate a long-run cointegration and empirical evidence testifies a leader-follower pattern between the two central banks. According to this pattern, the Bank of England does follow the European Central Bank.

Keywords: Monetary policy; Interbank rates; Interbank market; Cointegration; Dependence; Uncovered interest parity.

\footnotetext{
${ }^{1}$ María del Carmen González Velasco, Departamento de Dirección y Economía de la Empresa, Facultad de Ciencias Económicas y Empresariales, Universidad de León, Campus de Vegazana, s/n, 24071, León (España). Roque Brinckmann, Centro Sócio-Econômico, Campus da Trindade, Trindade-Florianópolis, 88040-900, SC-Brasil.
} 


\section{INTRODUCCIÓN}

El 1 de enero de 1999 se inicia la tercera fase de la Unión Económica y Monetaria (UEM), constituida por once países miembros de la Unión Europea a los que se suma Grecia en 2001, Eslovenia en 2007, Chipre y Malta en 2008 y Eslovaquia en 2009, mientras que Dinamarca, Suecia y el Reino Unido deciden no incorporarse a la UEM, que constituye la segunda zona económica más importante del mundo después de Estados Unidos y, por tanto, no pueden participar en la toma de decisiones de la política monetaria ni en la ejecución de las mismas. En este momento se fijan los tipos de cambio de forma irrevocable, se implanta el euro como moneda única y se crea el Banco Central Europeo (BCE), que asume la responsabilidad sobre la política monetaria única, cuyo fin primordial consiste en mantener la estabilidad de precios para contribuir a la mejora de las perspectivas económicas y del nivel de vida de los ciudadanos.

El mercado interbancario desempeña un papel crucial en la transmisión de las decisiones de política monetaria en la medida en que asegura la homogeneidad de la distribución de la liquidez del BCE y del nivel de los tipos de interés a corto plazo en toda la zona euro. El segmento al contado del mercado interbancario que más se ha desarrollado es el mercado de las operaciones sin garantías debido a su alto grado de actividad y de liquidez, se concentra mayoritariamente en el vencimiento a un día y utiliza como tipos de referencia el Eonia ${ }^{2}$ y el Euribor. Tanto el marco operativo como la estrategia de política monetaria del BCE tienen una función específica en la ejecución de la política monetaria. La estrategia determina el nivel de los tipos de interés del mercado monetario necesario para mantener la estabilidad de precios en el medio plazo, mientras que el marco operativo establece el procedimiento a utilizar para conseguir dicho nivel con los instrumentos de política monetaria disponibles.

Para conseguir una zona monetaria óptima en la UE las políticas monetarias deben converger. Suecia, Dinamarca y el Reino Unido no pertenecen a la UEM, pero los beneficios de la independencia de sus políticas monetarias han sido pequeños en los últimos diez años y deberían decrecer a medida que aumenta la integración monetaria, tal como señala Flam (2008). También Buiter (2008) argumenta que el Reino Unido es más vulnerable a una triple crisis financiera (crisis bancaria, monetaria y de deuda) si no pertenece a la UEM, debido a que es un país pequeño, con gran exposición al sector bancario y una moneda que no constituye una moneda de reserva global, y una capacidad fiscal limitada debido al gap de solvencia del sector bancario.

Por este motivo, en este artículo analizamos la integración y dependencia de las políticas monetarias del Banco de Inglaterra y del Banco Central Europeo, como responsables de la política monetaria de un país de la zona no euro y de la UEM, respectivamente. El horizonte temporal considerado es el comprendido entre el 1 de enero de 1999, en el que se crea la zona euro, y el 30 de septiembre de 2009, ambos inclusive y como proxy

${ }^{2}$ El Eonia se define como el índice medio del tipo del euro a un día, publicado por la Federación Bancaria Europea. Es la media ponderada de todos los préstamos a un día no garantizados, de acuerdo con la información facilitada por un panel compuesto por las entidades de crédito más activas en el mercado monetario. 
de la política monetaria se utiliza el más utilizado en la literatura, el tipo overnight del mercado interbancario (Libor a un día y Eonia) ya que esta variable ha adquirido gran importancia en las decisiones de política monetaria a partir de la creación de la UEM y el mayor número y volumen de operaciones realizadas en este mercado se lleva a cabo a vencimientos de un día. Además, el análisis a corto plazo permite evaluar la integración financiera de varias zonas monetarias. El estudio se estructura en los siguientes apartados: revisión de la literatura, metodología, análisis empírico y resultados obtenidos, conclusiones y bibliografía.

\section{REVISIÓN DE LA LITERATURA}

La convergencia de la política monetaria es muy importante para el establecimiento de una zona monetaria y ha sido uno de los temas de investigación desde finales de los ochenta. Algunos autores utilizan técnicas de series temporales que relacionan la convergencia de los tipos de interés con la estacionariedad o no estacionariedad de las variables. Karfakis y Moschos (1990), Katsimbris y Miller (1993) y Edison y Kole (1994) utilizan la cointegración para contrastar la convergencia de los tipos de interés de Alemania con otros países de la UEM y obtienen ausencia de cointegración para todos los países analizados. Fountas y Wu (1998) también utilizan la cointegración para contrastar la relación entre los tipos de interés a corto plazo de Alemania y seis países de la UEM y deducen la existencia de convergencia para cuatro de ellos. Camarero et al. (2002) analizan el proceso de convergencia seguido por los países de la Unión Europea (UE) para cumplir el criterio de tipos de interés fijado en el Tratado de Maastricht, aplican cointegración y deducen que existe convergencia para todos los países de la UE, excepto para Italia. Kiymaz y Waller (2002) utilizan la cointegración para contrastar la existencia de relaciones significativas en el largo plazo, recurren a los procedimientos de Johansen y al test de causalidad de Granger y encuentran un significativo impacto de los tipos de interés a corto plazo estadounidenses sobre los tipos de interés europeos. Galindo y Salcines (2003) efectúan un análisis de la presencia de raíces unitarias en la tasa de interés real del mercado interbancario, lo que indica la existencia de serios cambios estructurales en las series estudiadas, y concluyen indicando que el proceso de desregulación financiera, iniciada en la década de los ochenta, así como la liberación de los flujos de capitales internacionales, han conducido a una integración monetaria entre países, en la que Europa representa un papel primordial. Holtemoller (2005) estudia las desviaciones de la paridad no descubierta de tipos de interés para los nuevos países miembros entre 1994 y 2004, utiliza contrastes estadísticos recursivos y modelos de corrección de error y concluye indicando que Estonia y Lituania presentan el mayor grado de integración monetaria con la zona euro. Brada y Kutan (2001) comparan la convergencia con la política monetaria alemana de los países que próximamente van a pertenecer a la UE, los demás candidatos y los que recientemente se han incorporado a la UE entre 1993 y 2000, aplican la metodología de cointegración utilizando la base monetaria con periodicidad mensual y concluyen indicando que se observa convergencia entre la política monetaria de Alemania y los tres grupos de países, pero este vínculo es más débil en el caso de los candidatos. Brada et al. (2005) analizan la convergencia real y monetaria entre Alemania y los países más recientes de la UE, aplican la cointegración 
a la base monetaria, M2 y el índice de precios al consumo y demuestran que los países más recientes están cointegrados con los países que conforman el núcleo de la UE entre 1980 y 2000 . Kocenda et al. (2006) analizan la convergencia nominal y real entre diez países que se han incorporado recientemente a la UE y sus resultados indican que existe una convergencia fuerte entre tipos de interés e inflación. Sander y Kleimeier (2006) estudian la convergencia entre tipos de interés para ocho países que se han incorporado recientemente a la UE y sus resultados muestran evidencia de convergencia teniendo en cuenta como factores determinantes la concentración del mercado, la solvencia bancaria, la participación de la banca extranjera y el régimen de la política monetaria. Weber (2006) presenta un análisis de la convergencia entre la tasas de interés británica, europea y estadounidense, tanto en el corto como en el largo plazo, estudia las similitudes y diferencias entre distintas zonas monetarias, utiliza como variable de comparación la evolución de los tipos de interés, y logra encontrar un leve aumento de la convergencia entre los tipos europeos. De ello se puede deducir como economías con patrones monetarios diferentes pueden presentar o no similitudes relevantes $\mathrm{y}$, en su caso, deben ser mejor analizadas con el fin de obtener mejores conclusiones para la toma de decisiones en las inversiones globalizadas. Sørensen y Werner (2006) investigan la intensidad con que la política monetaria influye sobre las tasas aplicadas por el mercado regresando ciertos indicadores, algunos cíclicos como el PIB, sobre la velocidad de cambio de los ajustes obtenidos, y concluyen afirmando que el diferente grado de competencia existente entre los sectores bancarios de la zona euro constituye el principal factor explicativo de las diferencias entre las velocidades de ajuste presentadas entre los bancos de los diferentes mercados europeos analizados. Kasman et al (2008) analizan la convergencia entre las políticas monetarias de los trece países candidatos a incorporarse a la UE y recientes miembros de la UE y Alemania, utilizan el test de cointegración de Gregory y Hansen y sus resultados indican que la teoría de la paridad no descubierta de tipos de interés sólo se cumple para Estonia, Croacia y Turquía. Pesaran et al (2007) utiliza un modelo VAR global para estimar los efectos de una entrada hipotética del Reino Unido y Suecia en la zona euro. Buiter (2008) presenta varias razones para justificar la entrada de Suecia y de Inglaterra en la UME. Flam et al. (2008) indican que los beneficios de la independencia de las políticas monetarias han sido pequeños durante los últimos diez años y que decrecerán con el incremento de la integración financiera. Reade y Volz (2009a y 2009b) investigan el grado de independencia de la política monetaria, utilizan la metodología de VAR cointegrados, encuentran escasa evidencia de independencia de la política monetaria para países distintos de Estados Unidos, Japón y Alemania, también obtienen relaciones de cointegración entre todos los países miembros de la UEM, pero no entre el Reino Unido y Alemania, a pesar de su papel dominante en la zona euro. Por último, Reade y Volz (2009) utilizan la metodología de VAR cointegrados para investigar el grado de independencia de la política monetaria del Sveriges Riksbank y para ello consideran un par de países (Suecia y la zona euro), tipos de interés diarios del mercado interbancario entre enero de 1987 y junio de 2009 (Stibor y Euribor) y obtienen una relación de cointegración entre Suecia y la zona euro, que les lleva a aconsejar la incorporación de Suecia a la UEM, tras analizar también la convergencia de otras variables reales y financieras como el PIB, la inflación, los tipos de interés a largo plazo, la tasa de paro y el tipo de cambio de la corona sueca con respecto al euro. 
La mayoría de los estudios anteriores utilizan como proxy de la política monetaria los tipos de interés a corto plazo. Sin embargo, otros como MacDonald y Taylor (1991) utilizan la oferta monetaria con periodicidad mensual, Hafer y Kutan (1994) y Bredin (1994) consideran ambas variables, la base monetaria y los tipos de interés diarios para medir la política monetaria.

Nuestra aportación consiste en analizar la integración y dependencia entre las políticas monetarias de la zona euro y del Reino Unido desde la constitución de la UEM hasta septiembre de 2009, ambos inclusive, teniendo en cuenta como proxy de la política monetaria los tipos de interés diarios del mercado interbancario (Eonia y Libor overnight) con el fin de contrastar la hipótesis de dominio de la zona euro sobre el Reino Unido.

\section{METODOLOGÍA APLICADA}

La convergencia de las políticas monetarias de la UE se analiza contrastando la paridad descubierta de tipos de interés para el Reino Unido (zona no euro) y la UEM, que establece que la diferencia entre los tipos de interés nominales de ambas zonas debe ser igual a la evolución esperada del tipo de cambio más una prima de riesgo:

$$
r_{t}-\alpha-\beta r_{t}^{*}=E_{t}\left(s_{t+1}-s_{t}\right)+p_{t}
$$

donde:

$r_{t}$ : tipo de interés del Reino Unido (Libor overnight).

$r_{t}^{*}$ : tipo de interés de la UEM (Eonia).

$E_{t}\left(s_{t+1}-s_{t}\right)$ : esperanza de la evolución del logaritmo del tipo de cambio entre el Reino Unido y la UEM.

$p_{t}$ : prima de riesgo.

$\alpha, \beta$ : parámetros.

La prima de riesgo tiende a reducirse a medida que aumenta la integración financiera de modo que su evolución puede interpretarse como una medida de integración monetaria. Por tanto, el contraste de hipótesis de la teoría de la paridad descubierta de tipos de interés, teniendo en cuenta la hipótesis de las expectativas racionales, se basa en la siguiente expresión:

$$
r_{t}=\alpha+\beta r_{t}^{*}+\varepsilon_{t}
$$

donde $\varepsilon_{t}$ es el error de la regresión, que es estacionario.

Los tipos de interés del Reino Unido y de la UEM convergen si $\alpha=0$ y $\beta=1$. Para efectuar el contraste de la teoría de la paridad descubierta de tipos de interés la hipótesis 
nula $H_{0}: \beta=1$ indica que los cambios en el tipo de interés de la UEM se reflejan completamente en el tipo de interés del Reino Unido.

El método que se utiliza en el presente estudio es un análisis de cointegración para detectar el grado de integración de las políticas monetarias del Reino Unido y de la UEM y se consideran como medidas los tipos de interés diarios del mercado interbancario (Libor Interbank Offered Rate y Eonia Overnight Index Average). El horizonte temporal considerado se extiende desde enero de 1999, en que se crea la UEM, hasta el 30 de septiembre de 2009. Los datos han sido obtenidos de Bloomberg. tienen una periodicidad diaria y se expresan en porcentaje. El software utilizado es el Eviews, versión 7.

Para efectuar el análisis de cointegración seguimos las siguientes fases:

a) Utilización de un método analítico para identificar la no estacionariedad y presencia de raíz unitaria de las variables utilizadas. Para ello se observan los gráficos de las series y sus correlogramas con el fin de analizar su comportamiento a medida que aumentan los retardos. Adicionalmente se realiza uno de los contrastes clásicos de raíces unitarias, el test de Dickey-Fuller Aumentado (test ADF) seleccionando el criterio de información de Schwarz (SIC) para la inclusión de los retardos. El objetivo de esta fase consiste en determinar el orden de integración de las series.

b) Para que haya cointegración entre dos series una combinación lineal de ellas debe ser estacionaria. Una vez identificadas las características de cada serie se procede a aplicar el procedimiento de cointegracion de Engle y Granger (1987), que establece dos supuestos básicos para que dos o más series sean cointegradas: todas las variables del modelo deben ser integradas del mismo orden y una combinación lineal de las series debe tener un orden de integración menor que el orden de las series que originan tal combinación lineal. El segundo supuesto sugiere que la combinación lineal sea la serie de residuos resultante de la diferencia de las series utilizadas en el análisis. Además, para garantizar el equilibrio a largo plazo entre dos series se debe cumplir un tercer supuesto, que su distancia sea constante a lo largo del tiempo. Esto exige que los residuos sean integrados de orden cero, $I(0)$, y para que se cumpla el primer supuesto, las series deben ser integradas de orden uno, I(1). Una vez que se cumplen los tres supuestos anteriores se puede estimar la regresión con las variables en niveles ya que los residuos son estacionarios. Teniendo en cuenta estas consideraciones se especifican y estiman las relaciones de largo plazo entre el Eonia y el Libor y se reservan los residuos estimados para comprobar si están integrados de orden I(0). Posteriormente se realiza la regresión de la primera diferencia de los residuos estimados sobre su primer retardo y se aplican los mismos contrastes que para las series en niveles y en primeras diferencias. Entonces, la relación formal entre un modelo de corrección de error, que combina variables en niveles y en primeras diferencias, y las relaciones de cointegración la establece el "Teorema de Representación de Granger" (Granger y Weiss (1983), que constituye un pilar para modelizar series económicas no estacionarias.

c) Se aplica el contraste de Gregory-Hansen o test ADFGH (1996) sobre la ecuación de largo plazo y sus residuos para identificar posibles cambios estructurales. Los valores críticos del test ADF modificados para el contraste ADFGH son los siguientes (Gregory and Hansen, 1996, p.109): 


\begin{tabular}{|l|c|c|c|}
\hline \multicolumn{1}{|c|}{ ecuación/ nivel de significación } & $\mathbf{1 \%}$ & $\mathbf{5 \%}$ & $\mathbf{1 0 \%}$ \\
\hline Level shift & $-5,13$ & $-4,61$ & $-4,34$ \\
\hline Level shift with Trend & $-5,45$ & $-4,99$ & $-4,72$ \\
\hline Regime shift & $-5,47$ & $-4,95$ & $-4,68$ \\
\hline Tabla ADFGH & \\
\hline
\end{tabular}

La hipótesis nula se refiere a la no existencia de cointegración como en el test ADF, y la hipótesis alternativa es más extensa porque permite la cointegración bajo la posibilidad de que haya uno o varios cambios estructurales. Por tanto, se pueden establecer las siguientes combinaciones de resultados de los contrastes ADF y ADFGH:

c.1) Si en los dos tests se acepta la hipótesis nula se puede concluir que las variables no están cointegradas:

Test ADF acepta Ho $\rightarrow$ no hay relación de largo plazo

Test ADFGH acepta Ho $\rightarrow$ no están cointegradas, no hay vector de largo plazo.

c.2) Si en los dos tests se rechaza la hipótesis nula se puede deducir que existe cointegración, pero no se puede afirmar que hay un cambio estructural:

Test ADF rechaza $\mathrm{Ho} \rightarrow$ hay relación de largo plazo

Test ADFGH rechaza Ho $\rightarrow$ no están cointegradas, existe un vector de largo plazo, pero no se puede afirmar si ha habido un cambio estructural.

c.3) Si el test ADF rechaza la hipótesis nula y el test ADFGH la acepta se deduce que existe una relación de largo plazo y un vector de cointegración, que es estable para toda la muestra:

Test ADF rechaza $\mathrm{Ho} \rightarrow$ hay relación de largo plazo

Test ADFGH acepta Ho $\rightarrow$ existe un vector de cointegración de largo plazo.

c.4) Si el test ADF acepta la hipótesis nula y el test ADFGH la rechaza se puede concluir afirmando que existe cointegración con cambio estructural:

Test ADF acepta Ho $\rightarrow$ no hay relación de largo plazo

Test ADFGH rechaza Ho $\rightarrow$ existe cointegración con cambio estructural.

d) Finalmente, se aplica el contraste de cointegración de Johansen (1988) por estimación del VAR. Johansen recomienda especificar un VAR con las series integradas de orden uno, determinar el retardo óptimo del VAR para asegurar que los residuos sean ruido blanco, especificar las variables determinísticas como variables dummy, tendencias etc., y diagnosticar el VAR estimado. Después de la aplicación del procedimiento de máxima verosimilitud al VAR para determinación del rango de cointegración del sistema, se efectúa la estimación del modelo del Vector de Corrección de Errores (MCE) y consecuentemente se determina la relación causal entre las variables del modelo. Engle e Granger (1987) también demuestran que, si dos (o más) variables son cointegradas, es decir, existe una relación de equilibrio en el largo plazo no espuria, la mejor especificación de la relación en el corto y en el largo plazo es a través de un modelo de corrección de error. 


\section{ANÁLISIS EMPÍRICO Y RESULTADOS OBTENIDOS}

Las series consideradas para el análisis empírico en la relación de cointegración y su nomenclatura en el programa Eviews son las siguientes: Eonia (EONIA) y Libor (LIBOR). Se dispone en total de 3923 observaciones entre el 4 de enero de 1999 y el 30 de septiembre de 2009.

Se tienen en cuenta las fases indicadas en la metodología:

a) Para identificar la no estacionariedad y presencia de raíz unitaria de las variables utilizadas se analizan sus gráficos, sus correlogramas y se aplica el test ADF.

Figura 1

\section{Evoluc ión de los tipos de interés (en niveles)}
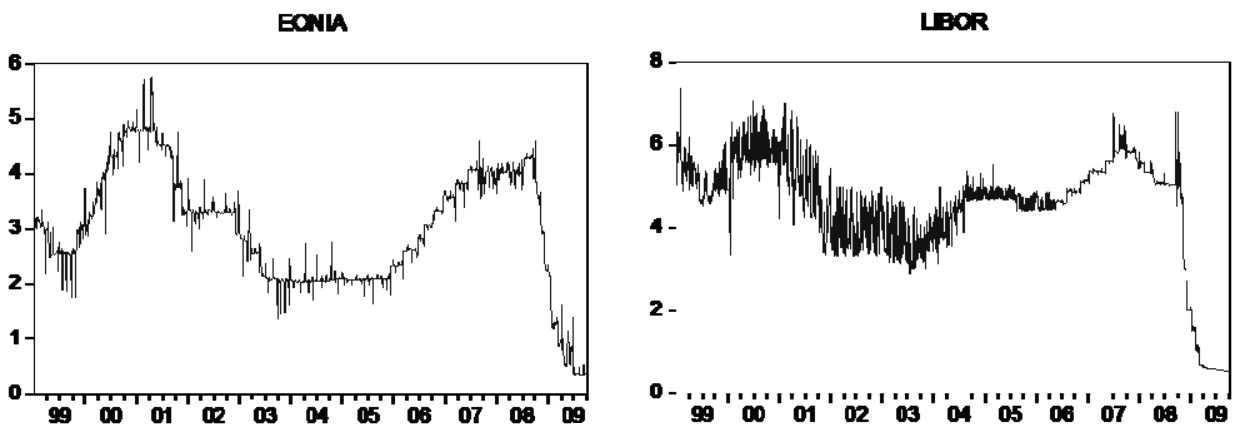

Figura 2

Comelograma del Eonia (en niveles)

Sample: 4/01/1999 30/09/2009

Included observations: 3923

\begin{tabular}{|c|c|c|c|c|c|c|}
\hline Autocomelation & Partial Comelation & & AC & PAC & Q-Stat & Prob \\
\hline 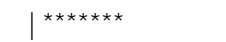 & 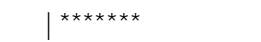 & 1 & 0.995 & 0.995 & 3884.9 & 0.000 \\
\hline 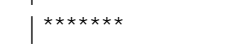 & |* | & 2 & 0.990 & 0.086 & 7737.3 & 0.000 \\
\hline 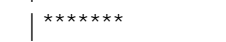 & $1 \quad 1$ & 3 & 0.987 & 0.073 & 11563. & 0.000 \\
\hline | *ktkk1kk & i & 4 & 0.983 & 0.030 & 15362. & 0.000 \\
\hline | & i & 5 & 0.981 & 0.060 & 19141. & 0.000 \\
\hline 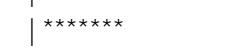 & i & 6 & 0.978 & 0.068 & 22902. & 0.000 \\
\hline 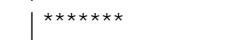 & | & 7 & 0.976 & 0.048 & 26649. & 0.000 \\
\hline I Fakdedek & i & 8 & 0.974 & 0.027 & 30381. & 0.000 \\
\hline 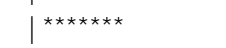 & | & 9 & 0.972 & 0.041 & 34102. & 0.000 \\
\hline 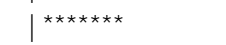 & i & 10 & 0.971 & 0.016 & 37809. & 0.000 \\
\hline 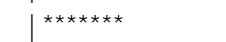 & i & 11 & 0.969 & 0.040 & 41506. & 0.000 \\
\hline 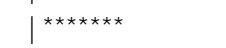 & i & 12 & 0.968 & 0.018 & 45193. & 0.000 \\
\hline
\end{tabular}




\section{Figura 3}

\section{Comelograma del Libor (en niveles)}

Sample: 4/01/1999 30/09/2009

Included observations: 3923

\begin{tabular}{|c|c|c|c|c|c|c|}
\hline Autocomelation & Partial Comelation & & AC & PAC & Q-Stat & Prob \\
\hline | *atedetcks & 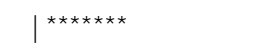 & 1 & 0.976 & 0.976 & 3737.0 & 0.000 \\
\hline | & |* | & 2 & 0.960 & 0.166 & 7355.1 & 0.000 \\
\hline | *atededk & |* $\mid$ & 3 & 0.949 & 0.129 & 10893. & 0.000 \\
\hline 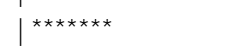 & * | & 4 & 0.941 & 0.096 & 14370. & 0.000 \\
\hline 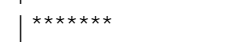 & * | & 5 & 0.935 & 0.089 & 17802. & 0.000 \\
\hline | Fexdedike & $1 \quad 1$ & 6 & 0.929 & 0.067 & 21198. & 0.000 \\
\hline 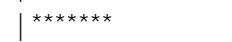 & $\left.\right|^{*} \mid$ & 7 & 0.926 & 0.081 & 24568. & 0.000 \\
\hline 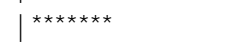 & $1 \quad 1$ & 8 & 0.921 & 0.023 & 27905. & 0.000 \\
\hline 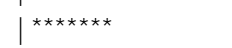 & i & 9 & 0.917 & 0.039 & 31213. & 0.000 \\
\hline 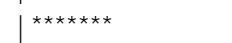 & | & 10 & 0.913 & 0.040 & 34495. & 0.000 \\
\hline * * * & I & 11 & 0.909 & 0.013 & 37748. & 0.000 \\
\hline | & $1 \quad 1$ & 12 & 0.905 & 0.025 & 40975. & 0.000 \\
\hline
\end{tabular}

Tal como se observa en las Figuras 1 a 3 podemos deducir que las variables objeto de estudio muestran el comportamiento característico de las series no estacionarias, donde las funciones de autocorrelación total comienzan en un valor muy próximo a $1(0,995$ en la del Eonia y 0,976 en la del Libor), que desciende suavemente y las funciones de autocorrelación parcial comienzan también con un primer valor muy próximo a 1 (los mismos valores que para las funciones de autocorrelación total) y el resto son aproximadamente cero. Para confirmar estos resultados, aplicamos el test ADF a las variables, objeto de análisis, que se encuentran expresadas en niveles (Figuras 4 a 5):

\section{Figura 4}

\section{Test ADF del Eonia (en niveles)}

Null Hypothesis: EONIA has a unit root

Exogenous: Constant

Lag Length: 8 (Automatic - based on SIC, maxlag=30)

\begin{tabular}{lccc}
\hline & t-Statistic & Prob.* \\
\hline Augmented Dic key-Fuller test sta tistic & -0.860158 & 0.8011 \\
\hline Test critic al values: & 1\% level & -3.431836 & \\
& 5\% level & -2.862082 & \\
& $10 \%$ level & -2.567102 & \\
\hline
\end{tabular}

*MacKinnon (1996) one-sided p-values. 
Augmented Dickey-Fuller Test Equation

Dependent Variable: D(EONIA)

Method: Least Squares

Sample (adjusted): 13/01/1999 30/09/2009

Included observations: 3914 a fter adjustments

\begin{tabular}{crcrr}
\hline Variable & Coefficient & Std. Enor & t-Statistic & Prob. \\
\hline EONIA(-1) & -0.001298 & 0.001509 & -0.860158 & 0.3898 \\
D(EONIA(-1)) & -0.146234 & 0.016035 & -9.119748 & 0.0000 \\
D(EONIA(-2)) & -0.120598 & 0.016183 & -7.451912 & 0.0000 \\
D(EONIA(-3)) & -0.073468 & 0.016248 & -4.521748 & 0.0000 \\
D(EONIA(-4)) & -0.100832 & 0.016201 & -6.223833 & 0.0000 \\
D(EONIA(-5)) & -0.102888 & 0.016196 & -6.352906 & 0.0000 \\
D(EO NIA(-6)) & -0.076582 & 0.016233 & -4.717804 & 0.0000 \\
D(EONIA(-7)) & -0.048562 & 0.016158 & -3.005476 & 0.0027 \\
D(EO NIA(-8)) & -0.055587 & 0.015999 & -3.474398 & 0.0005 \\
C & 0.002678 & 0.004798 & 0.558281 & 0.5767 \\
\hline R-squa red & 0.044354 & Mean dependent var & & -0.000679 \\
Adjusted R-squa red & 0.042151 & S.D. dependent var & & 0.101998 \\
S.E. of regresión & 0.099825 & Akaike info criterion & & -1.768248 \\
Sum squared resid & 38.90336 & Schwarz criterion & & -1.752222 \\
Log likelihood & 3470.460 & Hannan-Quinn criter. & & -1.762561 \\
F-statistic & 20.13263 & Durbin-Watson stat & & 2.002388 \\
Prob(F-statistic) & 0.000000 & &
\end{tabular}

\section{Figura 5 \\ Test ADF del Libor (en niveles)}

Null Hypothesis: LBOR has a unit root

Exogenous: Constant

Lag Length: 13 (Automatic - based on SIC, maxlag=30)

\begin{tabular}{lccc}
\hline & t-Statistic & Prob.* \\
\hline Augmented Dickey-Fuller test statistic & -1.303725 & 0.6301 \\
\hline Test critic al values: & 1\% level & -3.431838 & \\
& 5\% level & -2.862083 & \\
& $10 \%$ level & -2.567102 & \\
\hline
\end{tabular}

*MacKinnon (1996) one-sided p-values.

Augmented Dickey-Fuller Test Equation

Dependent Variable: $\mathrm{D}(\mathrm{UBOR})$

Method: Least Squares

6 Sample (adjusted): 18/01/1999 30/09/2009

( $)$ Included observations: 3909 after adjustments 


\begin{tabular}{|c|c|c|c|c|}
\hline Variable & Coefficient & Std. Enor & t-Statistic & Prob. \\
\hline LBOR(-1) & -0.004369 & 0.003351 & -1.303725 & 0.1924 \\
\hline D(LBOR(-1)) & -0.274427 & 0.016205 & -16.93477 & 0.0000 \\
\hline $\mathrm{D}($ UBOR(-2)) & -0.228936 & 0.016692 & -13.71513 & 0.0000 \\
\hline D(UBOR(-3)) & -0.197257 & 0.017032 & -11.58185 & 0.0000 \\
\hline D(UBOR(-4)) & -0.181474 & 0.017277 & -10.50408 & 0.0000 \\
\hline $\mathrm{D}($ UBOR(-5)) & -0.151841 & 0.017434 & -8.709682 & 0.0000 \\
\hline D(UBOR(-6)) & -0.151669 & 0.017510 & -8.662007 & 0.0000 \\
\hline D(UBOR(-7)) & -0.093374 & 0.017595 & -5.306902 & 0.0000 \\
\hline D(LBOR(-8)) & -0.102568 & 0.017468 & -5.871679 & 0.0000 \\
\hline D(UBOR(-9)) & -0.096565 & 0.017357 & -5.563634 & 0.0000 \\
\hline $\mathrm{D}($ UBO R(-10)) & -0.067876 & 0.017153 & -3.957092 & 0.0001 \\
\hline D(UBOR(-11)) & -0.077242 & 0.016853 & -4.583278 & 0.0000 \\
\hline $\mathrm{D}($ LBOR(-12)) & -0.102857 & 0.016462 & -6.248173 & 0.0000 \\
\hline $\mathrm{D}($ (பBOR(-13)) & -0.100554 & 0.015920 & -6.316309 & 0.0000 \\
\hline $\mathrm{C}$ & 0.015916 & 0.015723 & 1.012268 & 0.3115 \\
\hline R-squared & 0.114903 & \multicolumn{2}{|l|}{ Mean dependent var } & -0.001294 \\
\hline Adjusted R-squared & 0.111721 & \multicolumn{2}{|l|}{ S.D. dependent var } & 0.283842 \\
\hline S.E. of regresión & 0.267517 & \multicolumn{2}{|l|}{ Akaike info criterion } & 0.204566 \\
\hline Sum squared resid & 278.6763 & \multicolumn{2}{|l|}{ Schwarz criterion } & 0.228630 \\
\hline Log likelihood & -384.8240 & \multicolumn{2}{|l|}{ Hannan-Quinn criter. } & 0.213106 \\
\hline F-statistic & 36.10835 & \multicolumn{2}{|l|}{ Durbin-Watson stat } & 1.994191 \\
\hline Prob(F-statistic) & 0.000000 & & & \\
\hline
\end{tabular}

Los estadísticos del test ADF son mayores que los valores críticos fijados por MacKinnon (1996) para rechazar la hipótesis nula de existencia de una raíz unitaria al 1\%, 5\% y 10\% de significación en las series del Eonia y del Libor (-0,860158 para el Eonia y -1,303725 para el Libor), lo cual nos indica que no podemos rechazar la hipótesis nula y que las series tienen una raíz unitaria y, por tanto, no son estacionarias en niveles si incluimos un intercepto ya que el estadístico " $t$ " es sensible a las condiciones iniciales.

Se transforman las series en primeras diferencias y se aplican las mismas pruebas que a las series en niveles para identificar su estacionariedad o no estacionariedad: 
Figura 6

Evoluc ión de los tipos de interés (en primeras diferencias)
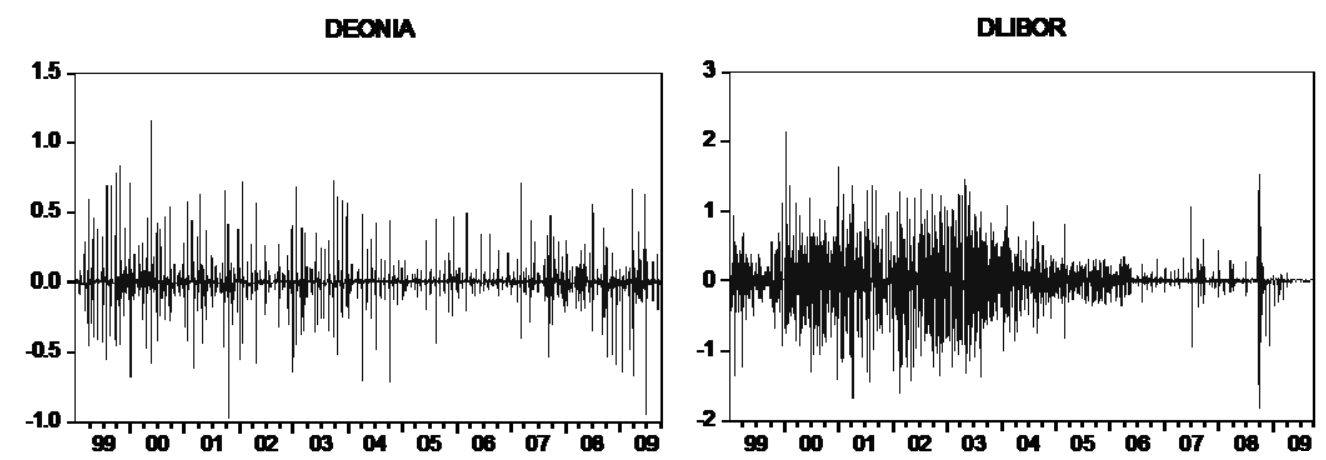

Figura 7

Comelograma del Eonia (en primeras differencias)

Sample: 4/01/1999 30/09/2009

Included observations: 3922

\begin{tabular}{|c|c|c|c|c|c|c|}
\hline Autoc omelation & Partial Comelation & & AC & PAC & Q-Stat & Prob \\
\hline I & $* \mid$ & 1 & -0.113 & -0.113 & 49.930 & 0.000 \\
\hline i & $* \mid$ & 2 & -0.077 & -0.091 & 73.307 & 0.000 \\
\hline I & I & 3 & -0.022 & -0.042 & 75.171 & 0.000 \\
\hline | & $* \mid$ & 4 & -0.059 & -0.075 & 88.667 & 0.000 \\
\hline i & $* \mid$ & 5 & -0.061 & -0.086 & 103.47 & 0.000 \\
\hline I & I & 6 & -0.030 & -0.065 & 107.01 & 0.000 \\
\hline i & i & 7 & -0.008 & -0.041 & 107.25 & 0.000 \\
\hline i & | & 8 & -0.027 & -0.056 & 110.12 & 0.000 \\
\hline l & I & 9 & 0.003 & -0.029 & 110.16 & 0.000 \\
\hline i & i & 10 & -0.025 & -0.055 & 112.68 & 0.000 \\
\hline i & i & 11 & -0.001 & -0.032 & 112.69 & 0.000 \\
\hline i & i & 12 & -0.013 & -0.041 & 113.33 & 0.000 \\
\hline
\end{tabular}




\section{Figura 8}

\section{Comelograma del Libor (en primeras diferencias)}

Sample: 4/01/1999 30/09/2009

Included observations: 3922

\begin{tabular}{|c|c|c|c|c|c|c|}
\hline Autoc omelation & Partial Comelation & & AC & PAC & Q-Stat & Prob \\
\hline | & $*$ & 1 & -0.185 & -0.185 & 134.36 & 0.000 \\
\hline i & $*$ & 2 & -0.104 & -0.143 & 176.90 & 0.000 \\
\hline I & $* \mid$ & 3 & -0.060 & -0.115 & 191.23 & 0.000 \\
\hline | & $* \mid$ & 4 & -0.047 & -0.107 & 199.99 & 0.000 \\
\hline i & $* \mid$ & 5 & -0.020 & -0.083 & 201.56 & 0.000 \\
\hline I & $* \mid$ & 6 & -0.038 & -0.098 & 207.14 & 0.000 \\
\hline i & i & 7 & 0.028 & -0.035 & 210.21 & 0.000 \\
\hline | & | & 8 & -0.011 & -0.052 & 210.69 & 0.000 \\
\hline I & I & 9 & -0.013 & -0.052 & 211.31 & 0.000 \\
\hline | & | & 10 & 0.015 & -0.023 & 212.14 & 0.000 \\
\hline | & | & 11 & -0.010 & -0.035 & 212.53 & 0.000 \\
\hline I & I & 12 & -0.045 & -0.077 & 220.59 & 0.000 \\
\hline
\end{tabular}

Las variables parecen moverse a lo largo del tiempo en torno a sus medias, varianzas y covarianzas y los valores de las funciones de autocorrelación son muy próximos a cero, comportamiento característico de las series estacionarias (Figuras 6 a 8). Por lo que respecta a los estadísticos del test $A D F$, se observa que son muy negativos y mucho menores que los valores críticos de Mackinnon (1996) para los niveles de significación del $1 \%, 5 \%$ y $10 \%$. En concreto, los valores para el test ADF son: $-28,60252$ para el Eonia y -25,98671 para el Libor, lo cual nos indica que se puede rechazar la hipótesis nula de existencia de una raíz unitaria y confirmar que las dos series son estacionarias si se expresan en primeras diferencias y no se incluyen variables exógenas (Figuras 9 y 10). Por tanto, se puede concluir que las series de los tipos de interés analizadas están integradas de orden I (1).

\section{Figura 9}

\section{Test ADF del Eonia (en primeras diferenc ias)}

Null Hypothesis: DEONIA has a unit root

Exogenous: Constant

Lag Length: 7 (Automatic - based on SIC, maxlag=30)

\begin{tabular}{lccc}
\hline & t-Statistic & Prob.* \\
\hline Augmented Dickey-Fuller test sta tistic & -28.60252 & 0.0000 \\
\hline Test critic al values: & 1\% level & -3.431836 & \\
& 5\% level & -2.862082 & \\
$10 \%$ level & -2.567102 & \\
\hline
\end{tabular}


*Mac Kinnon (1996) one-sided p-values. Augmented Dickey-Fuller Test Equation Dependent Variable: D(DEO NIA)

Method: Least Squares

Sample (adjusted): 1/13/1999 9/30/2009

Included obsenvations: 3914 after adjustments

\begin{tabular}{|c|c|c|c|c|}
\hline Variable & Coefficient & Std. Error & t-Statistic & Prob. \\
\hline DEONIA $(-1)$ & -1.731609 & 0.060540 & -28.60252 & 0.0000 \\
\hline$D(D E O N I A(-1))$ & 0.584284 & 0.055524 & 10.52318 & 0.0000 \\
\hline $\mathrm{D}(\mathrm{DEONIA}(-2))$ & 0.462648 & 0.049925 & 9.266841 & 0.0000 \\
\hline$D(D E O N I A(-3))$ & 0.388207 & 0.043960 & 8.830904 & 0.0000 \\
\hline $\mathrm{D}(\mathrm{DEONIA}(-4))$ & 0.286474 & 0.037892 & 7.560316 & 0.0000 \\
\hline$D(D E O N I A(-5))$ & 0.182761 & 0.031531 & 5.796185 & 0.0000 \\
\hline $\mathrm{D}(\mathrm{DEONIA}(-6))$ & 0.105415 & 0.024290 & 4.339767 & 0.0000 \\
\hline$D(D E O N I A(-7))$ & 0.056173 & 0.015984 & 3.514325 & 0.0004 \\
\hline $\mathrm{C}$ & -0.001213 & 0.001596 & -0.760058 & 0.4473 \\
\hline R-squared & 0.570374 & \multicolumn{2}{|l|}{ Mean dependent var } & 4.83E-05 \\
\hline Adjusted R-squared & 0.569494 & \multicolumn{2}{|l|}{ S.D. dependent var } & 0.152137 \\
\hline S.E. of regresión & 0.099822 & \multicolumn{2}{|l|}{ Akaike info criterion } & -1.768569 \\
\hline Sum squared resid & 38.91073 & \multicolumn{2}{|l|}{ Schwarzcriterion } & -1.754146 \\
\hline Log likelihood & 3470.090 & \multicolumn{2}{|l|}{ Hannan-Quinn criter. } & -1.763451 \\
\hline F-statistic & 648.0381 & \multicolumn{2}{|l|}{ Durbin-Watson stat } & 2.002455 \\
\hline Prob(F-sta tistic) & 0.000000 & & & \\
\hline
\end{tabular}

Null Hypothesis: $\mathrm{D}(\mathrm{LBOR})$ has a unit root

Exogenous: Constant

Lag Length: 12 (Automatic - based on SIC, maxlag=30)

\begin{tabular}{llll}
\hline & t-Statistic & Prob.* \\
\hline Augmented Dic key-Fuller test sta tistic & -25.98671 & 0.0000 \\
\hline Test critic al values: & 1\% level & -3.431838 & \\
& $5 \%$ level & -2.862083 & \\
& $10 \%$ level & -2.567102 & \\
\hline
\end{tabular}

*MacKinnon (1996) one-sided p-values.

Augmented Dickey-Fuller Test Equation

Dependent Variable: D(LBOR,2)

Method: Least Squares 
Sample (adjusted): 18/01/1999 30/09/2009

Included observations: 3909 after adjustments

\begin{tabular}{|c|c|c|c|c|}
\hline Variable & Coefficient & Std. Error & t-Sta tistic & Prob. \\
\hline D(UBOR(-1)) & -2.860293 & 0.110068 & -25.98671 & 0.0000 \\
\hline $\mathrm{D}(\operatorname{LBOR}(-1), 2)$ & 1.582046 & 0.105120 & 15.04996 & 0.0000 \\
\hline $\mathrm{D}(\mathrm{UBOR}(-2), 2)$ & 1.349526 & 0.099423 & 13.57363 & 0.0000 \\
\hline $\mathrm{D}(\mathrm{UBO} R(-3), 2)$ & 1.148876 & 0.093010 & 12.35221 & 0.0000 \\
\hline $\mathrm{D}($ UBOR(-4),2) & 0.964177 & 0.085904 & 11.22395 & 0.0000 \\
\hline $\mathrm{D}(\mathrm{UBO} R(-5), 2)$ & 0.809304 & 0.078310 & 10.33457 & 0.0000 \\
\hline $\mathrm{D}($ UBOR(-6),2) & 0.654788 & 0.070303 & 9.313819 & 0.0000 \\
\hline $\mathrm{D}(\mathrm{UBOR}(-7), 2)$ & 0.558746 & 0.061689 & 9.057451 & 0.0000 \\
\hline $\mathrm{D}($ UBOR(-8),2) & 0.453721 & 0.052989 & 8.562593 & 0.0000 \\
\hline $\mathrm{D}(\mathrm{UBO} R(-9), 2)$ & 0.354912 & 0.044037 & 8.059340 & 0.0000 \\
\hline $\mathrm{D}(\mathrm{UBOR}(-10), 2)$ & 0.285030 & 0.034959 & 8.153348 & 0.0000 \\
\hline $\mathrm{D}(\operatorname{UBOR}(-11), 2)$ & 0.206015 & 0.025673 & 8.024744 & 0.0000 \\
\hline $\mathrm{D}(\operatorname{LBOR}(-12), 2)$ & 0.101680 & 0.015898 & 6.395935 & 0.0000 \\
\hline $\mathrm{C}$ & -0.003808 & 0.004282 & -0.889358 & 0.3739 \\
\hline R-squared & 0.626580 & \multicolumn{2}{|l|}{ Mean dependent var } & 5.56E-19 \\
\hline Adjusted R-squared & 0.625334 & \multicolumn{2}{|l|}{ S.D. dependent var } & 0.437088 \\
\hline S.E. of regresión & 0.267541 & \multicolumn{2}{|l|}{ Akaike info criterion } & 0.204491 \\
\hline Sum squared resid & 278.7980 & \multicolumn{2}{|l|}{ Schwarzcriterion } & 0.226950 \\
\hline Log likelihood & -385.6769 & \multicolumn{2}{|l|}{ Hannan-Quinn criter. } & 0.212461 \\
\hline F-statistic & 502.7402 & \multicolumn{2}{|l|}{ Durbin-Watson stat } & 1.994356 \\
\hline Prob(F-statistic) & 0.000000 & & & \\
\hline
\end{tabular}

b) Se procede a aplicar el procedimiento de cointegración de Engle y Granger (1987) para comprobar si la serie del Libor está cointegrada con la del Eonia. Dado que las variables $L I B O R$ y EONIA resultaron estar integradas de orden I(1), se especifica y estima la relación de equilibrio a largo plazo entre ellas y se guardan los residuos estimados.

Se obtiene la siguiente ecuación de largo plazo que relaciona el Libor con el Eonia (Figura 11):

$$
\begin{aligned}
& \text { LIBOR }_{t}=\alpha+\beta \cdot \text { EONIA }_{t}+\widehat{z_{t}} \\
& \text { LIBOR }_{t}=1,875755+0,878823 \cdot \text { EONIA }_{t}+\widehat{z_{t}}
\end{aligned}
$$

El término error estimado por MCO a largo plazo es una medida del desequilibrio en el Eonia con respecto a su trayectoria de largo plazo:

$$
\hat{\mathrm{Z}}_{\mathrm{t}}=\mathrm{LIBOR}_{\mathrm{t}}-\hat{\alpha}-\hat{\beta} \cdot \mathrm{EONIA}_{\mathrm{t}}=\mathrm{LIBOR}_{\mathrm{t}}-1,875755-0,878823 \cdot \mathrm{EONIA}_{\mathrm{t}}
$$


Si se representan gráficamente los errores estimados se observa que los mismos varían en torno a la media, varianza y covarianzas, lo cual es un indicador característico de la estacionariedad (Figura 12).

Dependent Variable: $\amalg B O R$

Method: Least Squares

Sample: 1/04/1999 9/30/2009

Included observations: 3923

\begin{tabular}{lrlrr}
\hline \multicolumn{1}{c}{ Variable } & Coefficient & Std. Enor & t-Statistic & Prob. \\
\multicolumn{1}{c}{ C } & 1.875755 & 0.044430 & 42.21838 & 0.0000 \\
\multicolumn{1}{c}{ EONIA } & 0.878823 & 0.013976 & 62.87976 & 0.0000 \\
\hline R-squared & 0.502087 & Mean dependent var & 4.507474 \\
Adjusted R-squared & 0.501960 & S.D. dependent var & 1.323323 \\
S.E. of regresión & 0.933895 & Akaike info criterion & 2.701604 \\
Sum squared resid & 3419.740 & Schwarz criterion & 2.704803 \\
Log likelihood & -5297.197 & Hannan-Quinn criter. & & 2.702740 \\
F-statistic & 3953.865 & Durbin-Watson stat & & 0.102559 \\
Prob(F-statistic) & 0.000000 & & \\
\hline
\end{tabular}

Figura 12

Residuos de la relación de largo plazo entre el Libory el Eonia

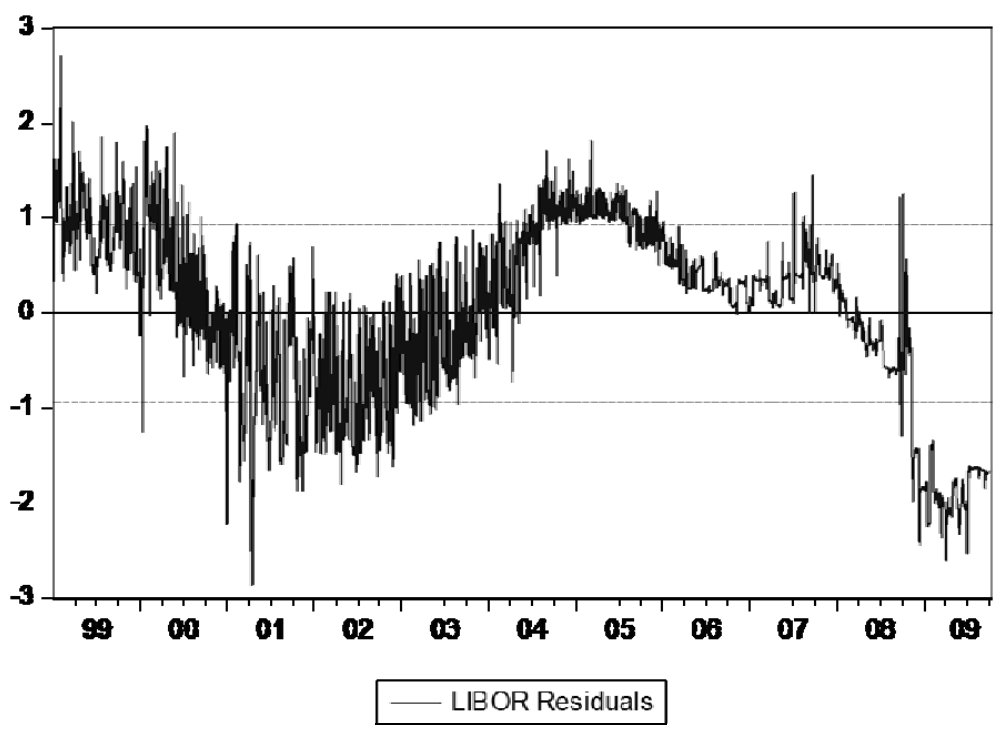


La metodología tradicional de la cointegración es aplicable a las series temporales sólo si los residuos estimados de la regresión son I(0) o ruido blanco. Para detectar si son estacionarios se estima la regresión de la primera diferencia de los residuos estimados sobre su primer retardo y los resultados muestran que el valor del parámetro $\alpha$ es estadísticamente significativo, incluso al $1 \%$ de significación ya que la probabilidad asociada al estadístico " $t$ " es inferior al nivel 0,01, con lo cual se rechaza la hipótesis nula de no cointegración a favor de la hipótesis de cointegración y se concluye que los residuos están integrados de orden $\mathrm{I}(0)$ teniendo en cuenta que no se ha incluido un intercepto porque los residuos proceden de una regresión con un término constante y, por tanto, $\left\{\widehat{z_{\mathrm{t}}}\right\}$ tiene media cero (Figura 13):

$$
\widehat{\Delta \mathrm{z}_{\mathrm{t}}}=\alpha \cdot \widehat{\mathrm{z}_{\mathrm{t}-1}}+\varepsilon_{\mathrm{t}}
$$

\section{Figura 13}

Regresión de la primera diferencia de los residuos de la relación de largo plazo entre el libory el Eonia sobre el primer retardo de los residuos

Dependent Variable: DRESILE

Method: Least Squares

Sample (adjusted): 1/05/1999 9/30/2009

Included obsenvations: 3922 after adjustments

\begin{tabular}{lcccr}
\hline \multicolumn{1}{c}{ Variable } & Coefficient & Std. Emor & t-Statistic & Prob. \\
\hline \multicolumn{1}{c}{ RESILE(-1) } & -0.051063 & 0.005051 & -10.10847 & 0.0000 \\
\hline R-squared & 0.025391 & Mean dependent var & -0.000788 \\
Adjusted R-squared & 0.025391 & S.D. dependent var & 0.299078 \\
S.E. of regresión & 0.295257 & Akaike info criterion & 0.398311 \\
Sum squared resid & 341.8188 & Schwarz criterion & 0.399911 \\
Log likelihood & -780.0877 & Hannan-Quinn criter. & 0.398879 \\
Durbin-Watson stat & 2.287135 & & \\
\hline
\end{tabular}

Posteriormente se ratifican estas conclusiones con el test de estacionariedad utilizado previamente para las series en niveles y en primeras diferencias ya que el valor del estadístico " $t$ " del test ADF en valor absoluto $(|-3,473377|)$ es mayor que los valores críticos fijados por Mackinnon (1996) al 1\%, 5\% y 10\% de significación, tal como se muestra en la Figura 14. Existe una relación a largo plazo estable, por lo que se puede afirmar que las variables $L I B O R$ y EONIA están cointegradas $\mathrm{CI}(1,1)$. 
Null Hypothesis: RESILE has a unit root

Exogenous: None

Lag Length: 13 (Automatic - based on SIC, maxlag=30)

\begin{tabular}{lccc}
\hline & t-Statistic & Prob.* \\
\hline Augmented Dic key-Fuller test statistic & -3.473377 & 0.0005 \\
\hline Test critic al values: & 1\% level & -2.565554 & \\
& 5\% level & -1.940905 & \\
& $10 \%$ level & -1.616645 & \\
\hline
\end{tabular}

*MacKinnon (1996) one-sided p-values.

Augmented Dickey-Fuller Test Equation

Dependent Variable: D(RESILE)

Method: Least Squares

Sample (adjusted): 1/18/1999 9/30/2009

Included observations: 3909 a fter a djustments

\begin{tabular}{crcrr}
\hline Variable & Coefficient & Std. Enor & t-Statistic & Prob. \\
\hline RESILE(-1) & -0.017950 & 0.005168 & -3.473377 & 0.0005 \\
D(RESILE(-1)) & -0.246836 & 0.016466 & -14.99062 & 0.0000 \\
D(RESILE(-2)) & -0.211151 & 0.016830 & -12.54587 & 0.0000 \\
D(RESILE(-3)) & -0.173872 & 0.017115 & -10.15918 & 0.0000 \\
D(RESILE(-4)) & -0.172412 & 0.017296 & -9.968079 & 0.0000 \\
D(RESILE(-5)) & -0.144046 & 0.017451 & -8.254466 & 0.0000 \\
D(RESILE(-6)) & -0.138688 & 0.017494 & -7.927725 & 0.0000 \\
D(RESILE(-7)) & -0.082166 & 0.017555 & -4.680598 & 0.0000 \\
D(RESILE(-8)) & -0.102425 & 0.017421 & -5.879535 & 0.0000 \\
D(RESILE(-9)) & -0.072953 & 0.017303 & -4.216352 & 0.0000 \\
D(RESILE(-10)) & -0.056723 & 0.017065 & -3.324031 & 0.0009 \\
D(RESILE(-11)) & -0.063329 & 0.016793 & -3.771234 & 0.0002 \\
D(RESILE(-12)) & -0.097139 & 0.016408 & -5.920066 & 0.0000 \\
D(RESILE(-13)) & -0.091129 & 0.015924 & -5.722646 & 0.0000 \\
\hline R-squa red & 0.110289 & Mean dependent var & & -0.000717 \\
Adjusted R-squared & 0.107319 & S.D. dependent var & & 0.298827 \\
S.E. of regression & 0.282337 & Akaike info criterion & & 0.312145 \\
Sum squa red resid & 310.4871 & Schwarzcriterion & & 0.334605 \\
Log likelihood & -596.0883 & Hannan-Quinn criter. & & 0.320116 \\
Durbin-Watson stat & 1.994986 & & & \\
\hline & & & & \\
\hline
\end{tabular}


A continuación se procede a relacionar el comportamiento a corto plazo de las variables LIBOR y EONIA con el comportamiento a largo plazo de las mismas a través del Mecanismo de Corrección de Errores propuesto inicialmente por Engle y Granger (1987). Dado que las variables EONIA y LIBOR están cointegradas, existe una relación de equilibrio a largo plazo entre ellas, pero en el corto plazo puede haber desequilibrio. El término error en la regresión de cointegración se interpreta como el error de equilibrio y sirve para relacionar el comportamiento de la variable $L I B O R$ con su valor a largo plazo. Se estima el modelo VAR en forma de vector de corrección de errores con el vector de cointegración normalizado con respecto a la variable LIBOR (Figura 15) y se muestran sus residuos (Figura 16), así como sus correlaciones (Figura 17):

\section{Figura 15}

Estimaciones del VAR en forma del vector de comección de emores (VARMCE)

Vector Error Correction Estimates

Sample (adjusted): 1/07/1999 9/30/2009

Included observations: 3920 after a djustments

Standard emors in ( ) \& t-sta tistic s in [ ]

\begin{tabular}{|c|c|c|}
\hline Cointegrating Eq: & CointEq1 & \\
\hline $\operatorname{LBOR}(-1)$ & 1.000000 & \\
\hline EONIA $(-1)$ & $\begin{array}{r}-1.000520 \\
(0.11176) \\
{[-8.95271]}\end{array}$ & \\
\hline $\mathrm{C}$ & -1.511170 & \\
\hline Emor Comection: & D(UBOR) & D(EONIA) \\
\hline CointEq 1 & $\begin{array}{r}-0.029164 \\
(0.00477) \\
{[-6.11103]}\end{array}$ & $\begin{array}{r}0.008367 \\
(0.00175) \\
{[4.79258]}\end{array}$ \\
\hline $\mathrm{D}(\mathrm{LBOR}(-1))$ & $\begin{array}{r}-0.194534 \\
(0.01601) \\
{[-12.1514]}\end{array}$ & $\begin{array}{r}-0.003728 \\
(0.00586) \\
{[-0.63651]}\end{array}$ \\
\hline $\mathrm{D}(\mathrm{LBOR}(-2))$ & $\begin{array}{r}-0.129940 \\
(0.01588) \\
{[-8.18097]}\end{array}$ & $\begin{array}{r}0.002653 \\
(0.00581) \\
{[0.45662]}\end{array}$ \\
\hline $\mathrm{D}(\mathrm{EONIA}(-1))$ & $\begin{array}{r}-0.064657 \\
(0.04342) \\
{[-1.48905]}\end{array}$ & $\begin{array}{r}-0.120836 \\
(0.01588) \\
{[-7.60731]}\end{array}$ \\
\hline
\end{tabular}


M.C. González Velasco y R. Brinckmann

D(EONIA(-2))

C $\begin{array}{rr}-0.022174 & -0.089210 \\ (0.04342) & (0.01588) \\ {[-0.51067]} & {[-5.61646]}\end{array}$

$\begin{array}{ll}-0.001653 & -0.000837\end{array}$

(0.00439) (0.00161)

[-0.37623] [-0.52063]

R-squared

Adj. R-squared

0.063734

0.026953

0.062538

0.025710

296.0142

39.61188

0.275008

53.28762

0.100601

21.68361

$-498.6979$

3443.408

0.257499

$-1.753780$

0.267102

$-1.744177$

$-0.001155$

$-0.000683$

0.284033

0.101920

0.000765

0.000763

2944.775

$-1.495293$

$-1.472887$

Figura 16

Residuos del VAR-MCE

LBOR Residuals

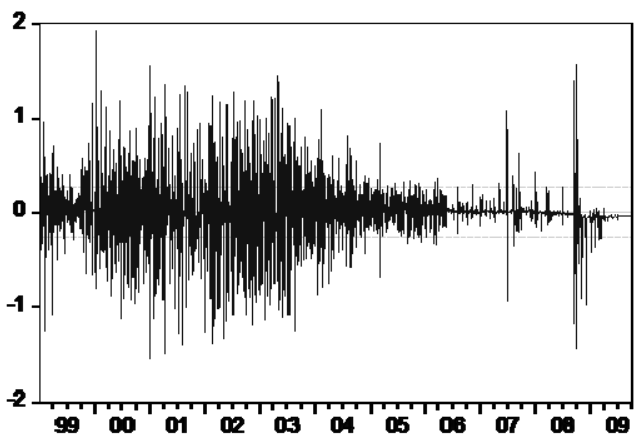

EONIA Residuals

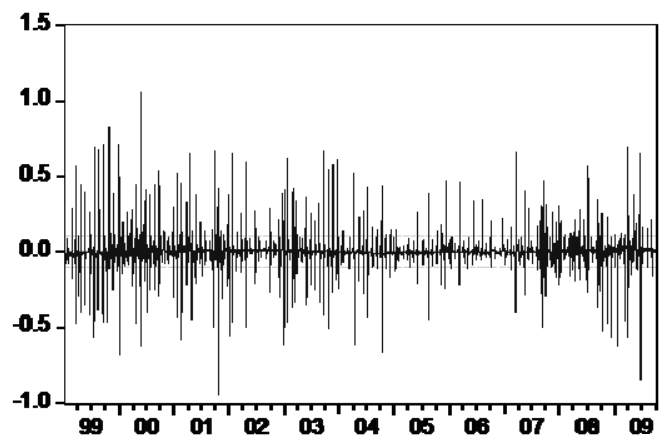


Autocorrelations with 2Std.Err. Bounds
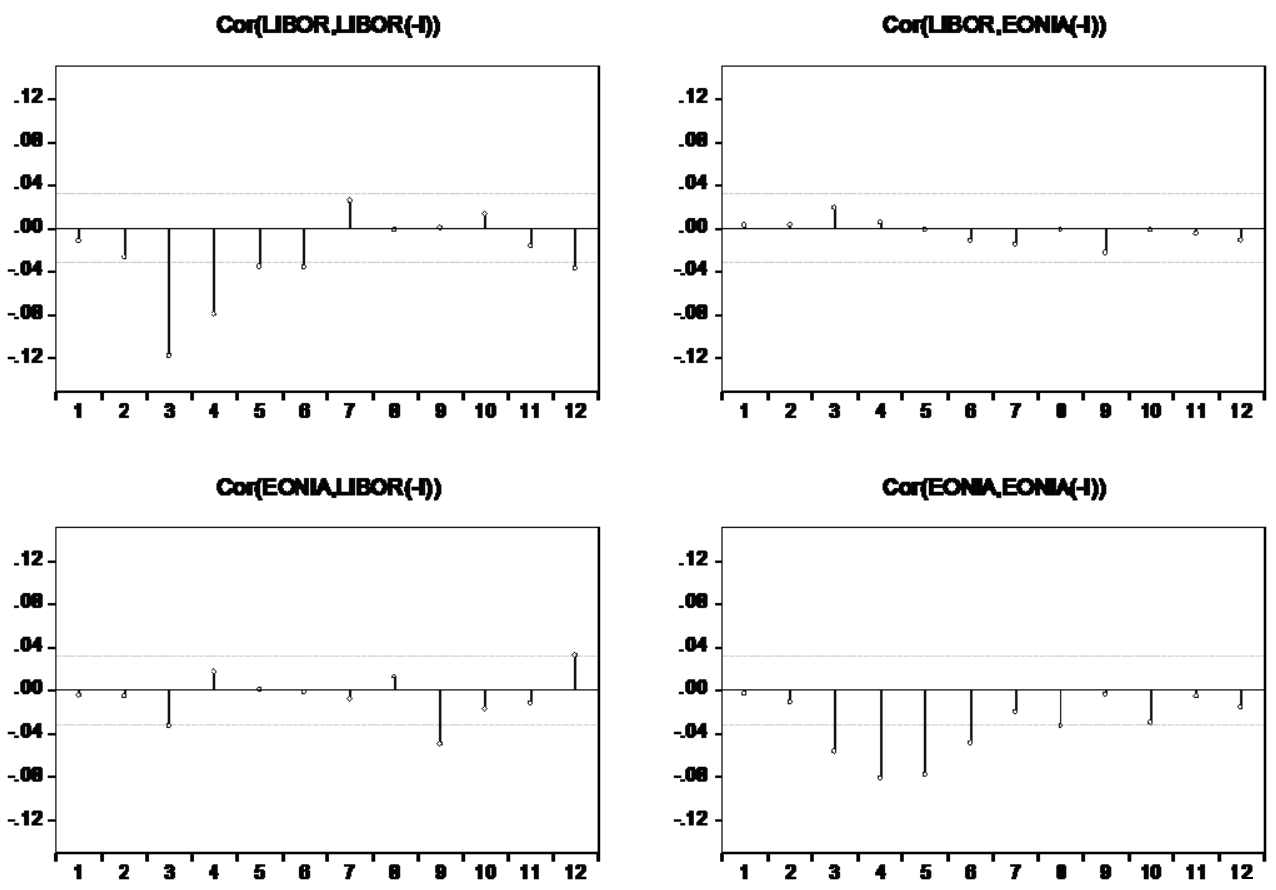

En la estimación del MCE se observan los parámetros significativos de la relación de largo plazo y los coeficientes de ajuste al equilibrio de largo plazo que determinan cuáles son las variables que se ajustan ante los desequilibrios en la relación de largo plazo. Teniendo en cuenta la siguiente representación del VAR-MCE:

$$
\left[\begin{array}{c}
\Delta L I B O R \\
\Delta E O N I A
\end{array}\right]=\left[\begin{array}{l}
\alpha_{1} \\
\alpha_{2}
\end{array}\right]\left[L I B O R-c-\beta L_{I B O R_{t-1}}\right]+\sum_{t=1}^{p} \Phi_{i}\left[\begin{array}{l}
\Delta \text { LIBOR }_{t-1} \\
\Delta E O N I A_{t-1}
\end{array}\right]+\left[\begin{array}{l}
a_{1 t} \\
a_{2 t}
\end{array}\right]
$$

los coeficientes de ajuste son $\alpha_{1}$ y $\alpha_{2}$. Según los resultados de la estimación se observa que sólo $\alpha_{1}=-0,029164$ es significativamente distinto de cero. Esto significa que el Eonia no reacciona a desvíos en la relación de equilibrio y, por tanto, puede considerarse como variable exógena y líder. Esto nos permite estimar el modelo VAR de forma uniecuacional sin perjuicio de las propiedades de los estimadores. Reflejamos la ecuación del Libor en primeras diferencias:

$$
\Delta \text { LIBOR }_{\mathrm{t}}=\mathrm{C}+\sum_{\mathrm{i}=1}^{12} \alpha_{\mathrm{t}-\mathrm{i}} \cdot \Delta \text { LIBOR }_{\mathrm{t}-\mathrm{i}}+\sum_{\mathrm{i}=1}^{12} \beta_{\mathrm{t}-\mathrm{i}} \cdot \Delta \text { EONIA }_{\mathrm{t}-\mathrm{i}}+\gamma \cdot \widehat{\mathrm{z}_{\mathrm{t}-1}}+\varepsilon_{\mathrm{t}}
$$

donde se refleja que la evolución de los cambios en la política monetaria de Inglaterra dependen de: 
- la persistencia de la política monetaria del Banco de Inglaterra, $\sum_{\mathrm{i}=1}^{12} \alpha_{\mathrm{t}-\mathrm{i}}$.

- las respuestas de la política monetaria del Reino Unido a las fluctuaciones en el corto plazo de la política monetaria del BCE, $\sum_{\mathrm{i}=1}^{12} \beta_{\mathrm{t}-\mathrm{i}}$.

- el coeficiente del término de corrección de error, $\gamma$.

y se obtiene la siguiente ecuación estimada (Figura 18):

$$
\begin{aligned}
& \Delta \text { LIBOR }_{t}=-0.00341454452958-0,00994784733316 \cdot E C M_{t-1}- \\
& -0,262070782602 \cdot \Delta L^{\prime B O R} t_{t-1}-\ldots-0,0746636348453 \cdot \Delta L I B O R_{t-12} \\
& -0,0527027840366 \cdot \Delta E O N I A_{t-1}-\ldots-0.0196964896198 \cdot \Delta E O N I A_{t-12}
\end{aligned}
$$

El coeficiente del término de corrección de error es -0,00994784733316 y tiene signo negativo para reducir el desequilibrio en el próximo periodo, en nuestro caso en el próximo día. Si las variables están en desequilibrio en el periodo "t-1", entonces el MCE actúa para restaurar las variables gradualmente hacia el equilibrio en el periodo " $t$ ", o en el futuro. La desviación del Libor respecto a su nivel de equilibrio a largo plazo se corrige diariamente un $1 \%$ aproximadamente, tal como indica el coeficiente del MCE, que se puede interpretar como la tasa de velocidad de ajuste en el corto plazo hasta alcanzar el equilibrio en el largo plazo.

\section{Mecanismo de comección de emores de la relación a largo plazo entre el Libory el Eonia}

Dependent Variable: DUBOR

Method: Least Squares

Sample (adjusted): 1/17/1999 9/30/2009

Included obsenvations: 3910 after adjustments

\begin{tabular}{ccccc}
\hline Variable & Coefficient & Std. Enor & t-Statistic & Prob. \\
\hline C & -0.003415 & 0.004307 & -0.792703 & 0.4280 \\
RESILE(-1) & -0.009948 & 0.004982 & -1.996778 & 0.0459 \\
DUBOR(-1) & -0.262071 & 0.016576 & -15.81043 & 0.0000 \\
DUBOR(-2) & -0.218374 & 0.017053 & -12.80537 & 0.0000 \\
DUBOR(-3) & -0.187025 & 0.017368 & -10.76815 & 0.0000 \\
DUBOR(-4) & -0.168959 & 0.017556 & -9.624045 & 0.0000 \\
DUBOR(-5) & -0.138716 & 0.017675 & -7.848188 & 0.0000 \\
DUBOR(-6) & -0.138914 & 0.017736 & -7.832338 & 0.0000 \\
DUBOR(-7) & -0.075282 & 0.017682 & -4.257533 & 0.0000 \\
DUBOR(-8) & -0.085228 & 0.017540 & -4.859001 & 0.0000
\end{tabular}




\begin{tabular}{|c|c|c|c|c|}
\hline DUBOR(-9) & -0.076073 & 0.017319 & -4.392393 & 0.0000 \\
\hline DUBOR(-10) & -0.046863 & 0.017040 & -2.750170 & 0.0060 \\
\hline DUBOR(-11) & -0.052694 & 0.016624 & -3.169779 & 0.0015 \\
\hline DUBOR(-12) & -0.074664 & 0.016037 & -4.655719 & 0.0000 \\
\hline DEONIA $(-1)$ & -0.052703 & 0.043348 & -1.215811 & 0.2241 \\
\hline DEONIA(-2) & -0.011430 & 0.043811 & -0.260902 & 0.7942 \\
\hline DEONIA $(-3)$ & 0.049974 & 0.044073 & 1.133886 & 0.2569 \\
\hline DEO NIA (-4) & 0.035424 & 0.044160 & 0.802179 & 0.4225 \\
\hline DEONIA $(-5)$ & 0.018479 & 0.044314 & 0.417000 & 0.6767 \\
\hline DEO NIA (-6) & -0.003345 & 0.044516 & -0.075147 & 0.9401 \\
\hline DEO NIA $(-7)$ & -0.009466 & 0.044525 & -0.212597 & 0.8317 \\
\hline DEONIA (-8) & 0.021908 & 0.044324 & 0.494274 & 0.6211 \\
\hline DEONIA $(-9)$ & -0.042564 & 0.044167 & -0.963703 & 0.3353 \\
\hline DEONIA (-10) & 0.005512 & 0.044053 & 0.125132 & 0.9004 \\
\hline DEONIA(-11) & -0.004897 & 0.043785 & -0.111849 & 0.9109 \\
\hline DEONIA(-12) & -0.019696 & 0.043303 & -0.454856 & 0.6492 \\
\hline & 0.107413 & \multicolumn{2}{|l|}{ Mean dependent var } & -0.001294 \\
\hline d R-squared & 0.101667 & \multicolumn{2}{|l|}{ S.D. dependent var } & 0.283806 \\
\hline egression & 0.268993 & \multicolumn{2}{|l|}{ Akaike info criterion } & 0.218362 \\
\hline uared resid & 281.0347 & \multicolumn{2}{|l|}{ Schwarz criterion } & 0.260063 \\
\hline lihood & -400.8973 & \multicolumn{2}{|l|}{ Hannan-Quinn criter. } & 0.233161 \\
\hline ic & 18.69577 & \multicolumn{2}{|l|}{ Durbin-Watson stat } & 2.014558 \\
\hline statistic ) & 0.000000 & & & \\
\hline
\end{tabular}

c) Se aplica el test de Gregory Hansen para detectar la presencia de uno o varios cambios estructurales y sus resultados nos permiten deducir que no se puede rechazar la hipótesis nula, lo que ratifica la existencia de una relación de largo plazo y de un vector de cointegración estable para toda la muestra sin un cambio estructural, aunque exista una sugerencia de una ruptura débil el 19 de febrero de 2008, quizás motivada por la crisis financiera internacional (Figura 19).

\section{Figura 19}

\section{Test de Gregory-Hansen}

\begin{tabular}{|cc|}
\hline & $\begin{array}{c}\text { The Gregory-Hansen Cointegration Test } \\
\text { Model 2: Level Shift } \\
\text { ADF Procedure }\end{array}$ \\
\hline t-stat & -2.600412 \\
Lag & 27.00000 \\
Break & $2 / 19 / 2008$ \\
\hline
\end{tabular}

d) Se aplica la metodología de cointegración de Johansen (1988) para contrastar si existen una o más ecuaciones de cointegración. Para ello se especifica un VAR con las series $L I B O R$ y EONIA que están integradas de orden I(1) con 96 retardos 
de las variables endógenas (Figura 20) y según los resultados de la estimación se observa también que sólo $\alpha_{1}=-0,682381$ es significativamente distinto de cero. Esto significa que el Eonia no reacciona a desvíos en la relación de equilibrio y, por tanto, puede considerarse como variable exógena y líder.

\section{Resultado parc ial del VAR de las variables UBOR y EONIA}

Vector Autoregression Estimates

Sample (adjusted): 4/10/1999 9/30/2009

Included observations: 3827 after a djustments

Standard emors in ( ) \& t-sta tistic s in [ ]

\begin{tabular}{|c|c|c|}
\hline & UBOR & EONIA \\
\hline UBOR(-1) & $\begin{array}{r}0.682381 \\
(0.01659) \\
{[41.1350]}\end{array}$ & $\begin{array}{r}0.003805 \\
(0.00629) \\
{[0.60514]}\end{array}$ \\
\hline UBOR(-2) & $\begin{array}{c}0.042437 \\
(0.02008) \\
{[2.11391]}\end{array}$ & $\begin{array}{r}0.008272 \\
(0.00761) \\
{[1.08703]}\end{array}$ \\
\hline UBOR(-3) & $\begin{array}{c}0.037841 \\
(0.02009) \\
{[1.88382]} \\
{[-0.46541]}\end{array}$ & $\begin{array}{r}-0.012380 \\
(0.00761) \\
{[-1.62595]} \\
{[-1.84785]}\end{array}$ \\
\hline பBOR(-96) & $\begin{array}{c}0.014670 \\
(0.01627) \\
{[0.90184]}\end{array}$ & $\begin{array}{r}-0.001994 \\
(0.00617) \\
{[-0.32332]}\end{array}$ \\
\hline $\operatorname{EONIA}(-1)$ & $\begin{array}{r}-0.032532 \\
(0.04375) \\
{[-0.74364]}\end{array}$ & $\begin{array}{r}0.820912 \\
(0.01658) \\
{[49.5040]}\end{array}$ \\
\hline EONIA (-2) & $\begin{array}{c}0.032849 \\
(0.05657) \\
{[0.58067]}\end{array}$ & $\begin{array}{r}0.029554 \\
(0.02144) \\
{[1.37825]}\end{array}$ \\
\hline EONIA $(-3)$ & $\begin{array}{c}0.057143 \\
(0.05659) \\
{[1.00985]}\end{array}$ & $\begin{array}{r}0.042065 \\
(0.02145) \\
{[1.96113]}\end{array}$ \\
\hline EONIA (-96) & $\begin{array}{r}-0.011787 \\
(0.04353) \\
{[-0.27076]}\end{array}$ & $\begin{array}{r}0.016834 \\
(0.01650) \\
{[1.02014]}\end{array}$ \\
\hline
\end{tabular}


C

$\begin{array}{rr}0.077530 & -0.006864 \\ (0.02043) & (0.00774) \\ {[3.79512]} & {[-0.88633]}\end{array}$

R-squared 0.963888

0.992193

Adj. R-squared

0.961981

0.991781

Sum sq. resids

242.4378

34.83527

S.E. equation

0.258290

0.097908

F-statistic

505.2015

2405.531

Log likelihood

$-150.7566$

3561.653

Akaike AIC

0.179648

$-1.760467$

Schwarz SC

0.494834

$-1.445280$

Mean dependent

4.478551

2.993355

S.D. dependent

1.324661

1.079947

Deteminant resid covariance (dof adj.)

0.000639

Determinant resid covariance

0.000576

Log likelihood

3411.395

Akaike information criterion

$-1.581079$

Schwarz criterion

$-0.950706$

Se aplica el test de causalidad de Granger para analizar si una variable endógena puede ser considerada como exógena. Según el estadístico de Wald se rechaza la hipótesis nula de no causalidad del Eonia sobre el Libor y se acepta la hipótesis alternativa de que la historia pasada de cambios en la política monetaria del BCE ha influido en el cambio de la política monetaria del Banco de Inglaterra si se consideran 96 retardos o más, pero no viceversa (Figura 21). Posteriormente se selecciona la longitud óptima del retardo considerando varios criterios de información (LR, FPE, AIC, SC y HQ) y finalmente se escoge el número de retardos que minimiza el Criterio de Información de Schwarz (SC), 7 retardos (Figura 22).

\section{Figura 21}

\section{Test de causalidad de Granger}

VAR Granger Causa lity/Block Exogeneity Wald Tests

Sample: 1/04/1999 9/30/2009

Included observations: 3827

Dependent variable: $U B O R$

\begin{tabular}{cccc}
\hline Excluded & Chi-sq & df & Prob. \\
\hline EONIA & 169.1857 & 96 & 0.0000 \\
\hline All & 169.1857 & 96 & 0.0000 \\
\hline
\end{tabular}


Dependent variable: EONIA

\begin{tabular}{cccc}
\hline Excluded & Chi-sq & df & Prob. \\
\hline UBOR & 118.7733 & 96 & 0.0575 \\
\hline All & 118.7733 & 96 & 0.0575 \\
\hline
\end{tabular}

\section{Selección de la longitud óptima del retardo}

VAR Lag Order Selection Criteria Endogenous variables: UBOR EONIA Exogenous variables: $\mathrm{C}$ Sample: 1/04/1999 9/30/2009 Included observations: 3827

\begin{tabular}{ccccccc}
\hline Lag & LogL & LR & FPE & AIC & SC & HQ \\
\hline 0 & -10857.14 & NA & 0.999260 & 5.675014 & 5.678280 & 5.676174 \\
1 & 2770.611 & 27234.13 & 0.000808 & -1.444793 & -1.434994 & -1.441312 \\
2 & 2852.834 & 164.2307 & 0.000776 & -1.485672 & -1.469341 & -1.479870 \\
3 & 2898.978 & 92.12006 & 0.000759 & -1.507697 & -1.484834 & -1.499574 \\
4 & 2927.515 & 56.93949 & 0.000749 & -1.520520 & -1.491125 & -1.510077 \\
5 & 2957.841 & 60.47739 & 0.000739 & -1.534278 & -1.498350 & -1.521514 \\
6 & 2981.848 & 47.85105 & 0.000731 & -1.544734 & -1.502274 & -1.529649 \\
7 & 3003.953 & 44.03660 & 0.000725 & -1.554196 & $-1.505203 *$ & -1.536790 \\
$\ldots \ldots$ & & & & & & \\
96 & & & & & & \\
\hline
\end{tabular}

* indic a tes lag order selected by the criterion

LR: sequential modified LR test statistic (each test at 5\% level)

FPE: Final prediction error

AIC: Aka ike information criterion

SC: Schwarz information criterion

HQ: Hannan-Quinn information criterion

Seguidamente se efectúa un diagnóstico de los residuos estimados en el VAR: se acepta la hipótesis nula de ausencia de correlación porque más del 95\% de las barras del correlograma se encuentran dentro del intervalo de confianza (Figura 23). 
Autocorrelations with 2 Std.Er. Bounds
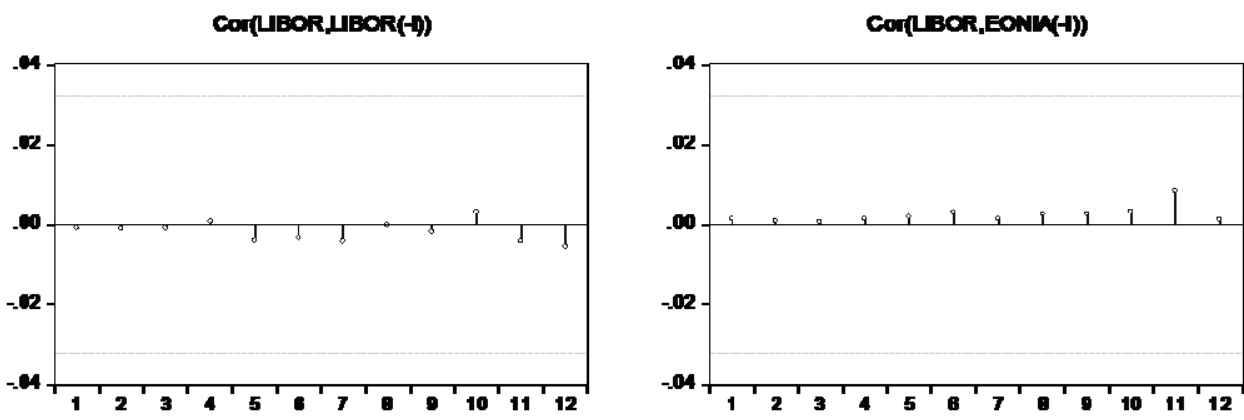

$\operatorname{Cor}($ ECNINUBOR(-4)

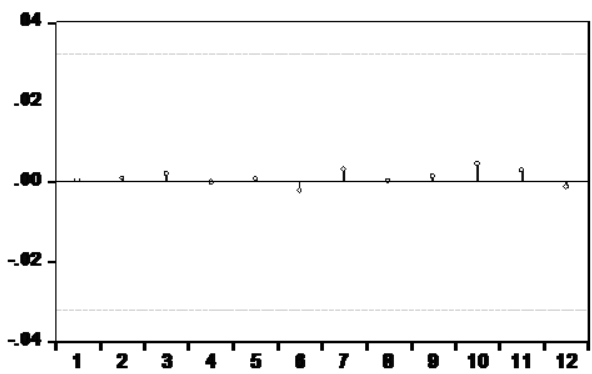

Cor(EONIAEONAN(-1))

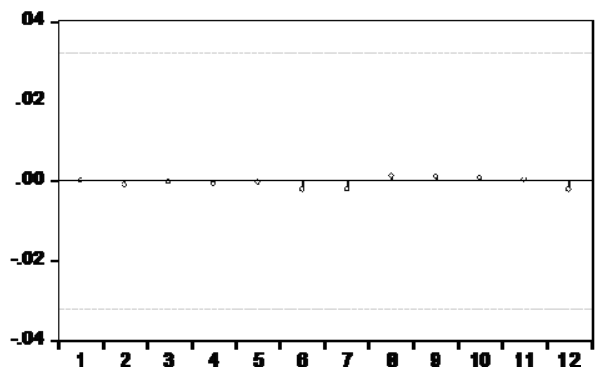

Siguiendo la metodología de Johansen se reformula el VAR en un Vector de Corrección de Errores (VEC), tal que:

$$
\Delta \mathrm{X}_{\mathrm{t}}=\Gamma_{1} \Delta \mathrm{X}_{\mathrm{t}-1}+\ldots+\Gamma_{\mathrm{k}-1} \Delta \mathrm{X}_{\mathrm{t}-(\mathrm{k}-1)}+\Pi \mathrm{X}_{\mathrm{t}-\mathrm{k}}+\mu+\varepsilon_{\mathrm{t}}
$$

donde:

$\mathrm{X}_{\mathrm{t}}$ es el vector de las variables endógenas integradas de orden I(1), LIBOR y EONIA.

$\mathrm{X}_{\mathrm{t}}=\sum_{\mathrm{i}=1}^{\mathrm{k}-1} \mathrm{~A}_{\mathrm{i}} \mathrm{X}_{\mathrm{t}-\mathrm{i}}+\mu+\varepsilon_{\mathrm{t}}$

$\Gamma_{\mathrm{i}}=\left(-\mathrm{I}+\mathrm{A}_{1}+\ldots+\mathrm{A}_{\mathrm{p}}\right) \quad \mathrm{I}=1,2, \ldots, \mathrm{k}-1$

$\Pi=\left(I-A_{1}-\ldots-A_{k}\right)$

$\Pi$ es una matriz $(\mathrm{N} \times \mathrm{N})$ de la forma $\Pi=\alpha \beta^{T}$ donde $\alpha$ y $\beta$ son matrices $(\mathrm{N} \times \mathrm{N})$ y $\varepsilon_{\mathrm{t}}$ es un vector $(\mathrm{N} \times 1)$ de términos de errores normal e independientemente distribuidos. 
$\alpha$ se puede interpretar como la velocidad de ajuste de cada variable para recuperar la posición de equilibrio en el largo plazo cuando se produzcan desviaciones de dicho equilibrio.

$\beta$ se refiere a las " $r$ " relaciones de cointegración (Figura 24)

Sample: 1/04/1999 9/30/2009

Included observations: 3915

Series: LBOR EONIA

Lags interval: 1 to 7

Selected ( 0.05 level*) Number of Cointegrating Relations by Model

\begin{tabular}{cccccc}
\hline Data Trend: & None & None & Linear & Linear & Quadratic \\
\hline Test Type & No Intercept & Intercept & Intercept & Intercept & Intercept \\
& No Trend & No Trend & No Trend & Trend & Trend \\
Trace & 1 & 1 & 1 & 1 & 1 \\
Max-Eig & 1 & 1 & 1 & 1 & 1 \\
\hline
\end{tabular}

*Critic al va lues based on Mac Kinnon-Haug-Mic helis (1999)

Information Criteria by Rank and Model

\begin{tabular}{|c|c|c|c|c|c|}
\hline Data Trend: & None & None & Linear & Linear & Quadratic \\
\hline Rank or & No Intercept & Intercept & Intercept & Intercept & Intercept \\
\hline No. of CES & No Trend & No Trend & No Trend & Trend & Trend \\
\hline \multicolumn{6}{|c|}{ Log Likelihood by Rank (rows) and Model (columns) } \\
\hline 0 & 3039.684 & 3039.684 & 3040.154 & 3040.154 & 3040.847 \\
\hline 1 & 3052.644 & 3056.879 & 3057.326 & 3057.671 & 3058.229 \\
\hline 2 & 3053.306 & 3057.687 & 3057.687 & 3059.014 & 3059.014 \\
\hline \multicolumn{6}{|c|}{ Aka ike Information Criteria by Rank (rows) and Model (columns) } \\
\hline 0 & -1.538536 & -1.538536 & -1.537754 & -1.537754 & -1.537087 \\
\hline 1 & -1.543113 & $-1.544766 *$ & -1.544483 & -1.544149 & -1.543923 \\
\hline 2 & -1.541408 & -1.542624 & -1.542624 & -1.542280 & -1.542280 \\
\hline \multicolumn{6}{|c|}{ Schwarz Criteria by Rank (rows) and Model (columns) } \\
\hline 0 & $-1.493674 *$ & $-1.493674 *$ & -1.489689 & -1.489689 & -1.485817 \\
\hline 1 & -1.491843 & -1.491893 & -1.490009 & -1.488072 & -1.486244 \\
\hline 2 & -1.483729 & -1.481741 & -1.481741 & -1.478193 & -1.478193 \\
\hline
\end{tabular}


El cuadro resumen indica una sola ecuación de cointegración tanto en la Prueba de la Traza como en la Prueba del Valor Propio Máximo en las cinco opciones posibles de tendencia, pero seleccionamos la segunda opción que se refiere a la existencia de intercepto en la ecuación de cointegración y no tendencia en el VAR (Figura 25).

\section{Figura 25}

\section{Test de cointegración de Johansen (opción 2)}

Sample (adjusted): 1/12/1999 9/30/2009

Included observations: 3915 after a djustments

Trend assumption: No deteministic trend (restric ted constant)

Series: UBOR EONIA

Lags interval (in first differences): 1 to 7

Unrestric ted Cointegration Rank Test (Trace)

\begin{tabular}{ccccc}
\hline $\begin{array}{c}\text { Hypothesized } \\
\text { No. of CE(s) }\end{array}$ & Eigenvalue & $\begin{array}{c}\text { Trace } \\
\text { Statistic }\end{array}$ & $\begin{array}{c}\mathbf{0 . 0 5} \\
\text { Critical Value }\end{array}$ & Prob.** \\
\hline None ${ }^{*}$ & 0.008746 & 36.00617 & 20.26184 & 0.0002 \\
At most 1 & 0.000413 & 1.615935 & 9.164546 & 0.8522 \\
\hline
\end{tabular}

Trace test indic ates 1 cointegrating eqn(s) at the 0.05 level

* denotes rejection of the hypothesis at the 0.05 level

**Mac Kinnon-Haug-Mic helis (1999) p-values

Unrestric ted Cointegration Rank Test (Maximum Eigenvalue)

\begin{tabular}{ccccc}
\hline $\begin{array}{c}\text { Hypothesized } \\
\text { No. of CE(s) }\end{array}$ & Eigenvalue & $\begin{array}{c}\text { Max-Eigen } \\
\text { Statistic }\end{array}$ & $\begin{array}{c}\mathbf{0 . 0 5} \\
\text { Critical Value }\end{array}$ & Prob.** \\
\hline None $*$ & 0.008746 & 34.39024 & 15.89210 & 0.0000 \\
At most 1 & 0.000413 & 1.615935 & 9.164546 & 0.8522 \\
\hline
\end{tabular}

Max-eigenvalue test indic ates 1 cointegrating eqn(s) at the 0.05 level

$*$ denotes rejection of the hypothesis at the 0.05 level

**Mac Kinnon-Haug-Mic helis (1999) p-values

Unrestric ted Cointegrating Coeffic ients (nomalized by $b^{*} * S 11 * b=1$ ):

\begin{tabular}{ccc}
\hline UBOR & EONIA & C \\
-1.123965 & 1.094754 & 1.829394 \\
-0.114862 & -0.523661 & 1.344852 \\
\hline
\end{tabular}

Unrestric ted Adjustment Coefficients (alpha):

$\begin{array}{lrl}\text { D(LBOR) } & 0.014200 & 0.004535 \\ \mathrm{D}(\text { EONIA }) & -0.007773 & 0.001121\end{array}$




\begin{tabular}{|c|c|c|c|}
\hline 1 Cointegrating & $n(s):$ & Log likelihood & 3056.879 \\
\hline Nomalized coir & g coefficie & ndard error in & eses) \\
\hline UBOR & EONIA & C & \\
\hline 1.000000 & -0.974011 & -1.627626 & \\
\hline & $(0.14331)$ & $(0.45566)$ & \\
\hline Adjustment coe & (standard & parentheses) & \\
\hline $\mathrm{D}(\mathrm{UBOR})$ & -0.015960 & & \\
\hline & $(0.00485)$ & & \\
\hline $\mathrm{D}(\mathrm{EONIA})$ & 0.008737 & & \\
\hline & (0.00179) & & \\
\hline
\end{tabular}

Los resultados de la Prueba de la Traza y de la Prueba del Valor Propio Máximo indican que se rechaza la hipótesis nula de no cointegración ya que los estadísticos de ambas pruebas son mayores que los valores críticos de MacKinnon-Haug-Michelis (1999) al 5\% de significación:

$36,00617>20,26184$ (Prueba de la Traza)

34,39024 > 15,89210 (Prueba del Valor Propio Máximo)

También se obtienen los coeficientes normalizados del vector de cointegración, que se puede interpretar como una función que relaciona las variables LIBOR y EONIA:

$$
\mathrm{LIBOR}_{\mathrm{t}}=-1,627626+0,974011 \cdot \mathrm{EONIA}_{\mathrm{t}}
$$

\section{CONCLUSIONES}

En este estudio se contrasta la interdependencia entre las políticas monetarias de la UEM y de la zona no euro (Reino Unido). Para ello se considera como proxy los tipos overnight del mercado interbancario (Eonia y Libor, respectivamente) porque constituyen variables de gran importancia en las decisiones de política monetaria del BCE y del Banco de Inglaterra, y porque el mayor volumen y liquidez de operaciones en el mercado interbancario se concentra en las operaciones con vencimiento a un día. Se aplica la metodología de cointegración de Engle y Granger (1987) y de Johansen (1991 y 1995) y se llega a la conclusión de que las políticas monetarias del ECB y del Banco de Inglaterra se encuentran cointegradas, lo que indica la existencia de una relación de equilibrio a largo plazo entre ambas, desempeñando además la primera el papel de líder. Se deduce del análisis que el Libor depende del Eonia y de su evolución en el pasado si se tienen en cuenta 96 ó más retardos, aproximadamente tres meses. También se observa un cambio estructural en febrero de 2008 motivado por la actual crisis económica y financiera, pero que no influye en la relación de cointegración entre ambas variables. 
Esto nos lleva a afirmar que el pertenecer a la UEM conlleva más ventajas que inconvenientes ya que la política monetaria del BCE es la que influye en las políticas monetarias de la zona no euro. Por este motivo apoyamos los argumentos de Buiter (2008) cuando indica que el Reino Unido es más vulnerable a una triple crisis financiera (crisis bancaria, monetaria y de deuda) si no pertenece a la UEM, debido a que es un país pequeño, con gran exposición al sector bancario y una moneda que no constituye una moneda de reserva global, y una capacidad fiscal limitada debido al gap de solvencia del sector bancario.

\section{REFERENCIAS BIBLIOGRÁFICAS}

Belke, A. y Cui, Y. (2009). "US-Euro area monetary policy interdependence. New evidence from Taylor Rule based VECMs", Ruhr Economic Papers, 85, Universität Duisburg-Essen.

Brada, J. y Kutan, A. (2001). "The convergence of monetary policy between candidate countries and the European Union”, Economic Systems, 25 (3), pp. 215-231.

Brada, J., Kutan, A. y Zhou, S. (2005). "Real and monetary convergence between the European Union's core and recent member countries: A rolling cointegration approach", Journal of Banking \& Finance, 29 (1), pp. 249-270.

Bredin, D. y Fountas, S. (1998). "Testing for monetary policy convergence in European countries", Journal of Economic Studies, 25 (5), pp. 353-369.

Buiter, W. (2008). "Why the United Kingdom should be join the eurozone", International Finance, 11 (3), pp. 269-282.

Chaboud, A. y Wright, J. (2005). “Uncovered interest parity: it works, but not for long”, Journal of International Economics, 66 (2), pp. 349-362.

Devine, M. (1997). "The cointegration of international interest rates: A review", Technical Paper, $n^{\circ}$ 1/RT/97, Economic Analysis, Research and Publications Department, Central Bank of Ireland.

Dickey, D.A. y Fuller, W.A. (1979). "Distribution of the estimators for autoregressive time series with a unit root", Journal of the American Statistical Association, 74 (366), pp.427-431.

Dickey, D.A. y Fuller, W.A. (1981). “Likelihood ratio statistics for autoregressive time series with a unit root”, Econometrica, 49 (4), pp. 1057-1072.

Dolado, J.; Gonzalo, J. y Marmol, F. (2001). "A primer in cointegration”. In, A companion to theoretical econometrics, Baltagi, B.H. (Ed.), chapter 30. New York: Blackwell.

Engle, R.F. y Granger, C.W.J. (1987). "Cointegration and error-correction: Representation, estimation and testing”, Econometrica, 55 (2), pp. 251-276. 
Engle, R. y Granger, C.W. (1987) “Cointegration and error correction: Representation, estimation and testing”, Econometrica, 55 (2), pp. 251-276.

Engle, R. y Granger, C.W. (1991). Long run economic relationships: Readings in cointegration, advanced texts in econometrics. Oxford: Oxford University Press.

Engle, R.F. y Yoo, B.S. (1987). "Forecasting and testing in cointegrated systems", Journal of Econometrics, 35 (1), pp. 143-159.

Engle, R.F. (2002). "Dynamic conditional correlation: a simple class of multivariate generalized autoregressive conditional heteroskedasticity models", Journal of Business and Economic Statistics, 20 (3), pp. 339-350.

Flam, H. et al. (2008). "EMU at ten. Should Denmark, Sweden and the UK join?, SNS Economic Policy Group Report, Stockholm.

Galindo, L.M. y Salcines, V. (2003). “Una nota sobre la hipótesis de Fisher en España en el proceso de convergencia europeo”, Momento Económico, 127, pp. 7277.

Gerdesmeier, D. y Roffia, B. (2004). "Taylor rules for the euro area: the issue of realtime data”, Discussion Paper Series, 1 (37), Deutsche Bundesbank, Research Centre.

Granger, C.W.J. (1969). "Investigating causal relations by econometric models and cross-spectral methods", Econometrica, 37 (3), pp. 424-438.

Granger, C.W.J. (2004). "Análisis de series temporales, cointegración y aplicaciones", Revista Asturiana de Economía, 30, pp. 197-206.

Granger, C.W.J. y Newbold, P. (1974). “Spurious regression in econometrics”, Journal of Econometrics, 2, (2), pp. 111-120.

Granger, C.W.J. y Weiss, A.A. (1983). "Time series analysis of error-correction models". In Studies in econometrics, time series and multivariate statistics, Karlin, S.; Amemiya, T. y Goodman, L.A. (Eds.). En honor de T.W. Anderson. San Diego: Academic Press, pp. 255-278.

Granger, C.W.J. y Lee, T.-H. (1990). “Multicointegration”. In Advances in econometrics: cointegration, spurious regressions and unit roots, Rhodes, G. F. y Fomby, T.B. (Eds.). New York: JAI Press, pp. 17-84.

Guisán, M.C. (2008). “Causalidad y cointegración en modelos econométricos: aplicaciones a los países de la OCDE y limitaciones de los tests de cointegración", Working Paper Series Economic Development, n. 61, Facultad de Ciencias Económicas y Empresariales, Universidad de Santiago de Compostela.

Hall, A.D.; Anderson, H.M. y Granger, C.W.J. (1992). "A cointegration analysis of treasury bill yields", Review of Economics and Statistics, 74 (1), pp. 116-126.

Hansen, P.R. y Johansen, S. (1998). Workbook on cointegration. Oxford: Oxford University Press. 
Hylleberg, S. et al. (1990). "Seasonal integration and cointegration", Journal of Econometrics, 44 (1-2), pp. 215-238.

Holtemoller, O. (2005). "Uncovered interest rate parity and analysis of monetary convergence of potential EMU accession countries", International Economics and Economic Policy, 2 (1), pp. 33-63.

Jaén García, M. y López Ruíz, E. (2001). Modelos econométricos de series temporales: teoría y práctica. Oviedo: Septem Ediciones.

Johansen, S. (1988) "Statistical analysis of cointegration vectors", Journal of Economic Dynamics and Control, 12 (2-3), pp. 231-254.

Johansen, S. y Juselius, K. (1990). "Maximum likelihood estimation and Inferences on cointegration with applications to the demand for money", Oxford Bulletin of Economics and Statistics, 52 (2), pp. 169-210.

Johansen, S. (1991). "Estimation and hypothesis testing of cointegration vectors in gaussian vector autoregressive models", Econometrica, 59 (6), pp. 15511580.

Johansen, S. (1995). Likelihood-based inference in cointegrated vector autoregressive models (Advanced tests in econometrics). Oxford: Oxford University Press.

Karfakis, J.C. y Demetrios, M.M. (1990). "Interest rate linkages within the European monetary system: A time series analysis", Journal of Money, Credit and Banking, vol. 22, $\mathrm{n}^{\circ}$ 3, pp. 388-394.

Kasman, A.; Kirbas-Kasman, S. y Turgutlu, E. (2008). “Monetary policy convergence of potential EMU accession countries: A cointegration analysis with shifting regimes", Economic Modelling, 25 (2), pp. 340-350.

Katsimbris, G. y Miller, S. (1993). "Interest rate linkages within the european monetary system: further analysis", Journal of Money, Credit and Banking, 25 (4), pp. 771-779.

Kiymaz, H. y Waller, E. (2002). "Cointegration between short-term international interest rates", WallerJournal of Accounting \& Finance Research, 12 (6), pp. 11-21.

Kocenda, E.; Kutan, A.M. y Yigit, T.M. (2006). "Pilgrims to the eurozone: how far, how fast?, Economic Systems, 30 (4), pp. 311-327.

Lardic, S. y Mignon, V. (2003). "Fractional cointegration between nominal interest rates and inflation: a re-examination of the fisher relationship in the G7 countries", Economics Bulletin, 3 (14), pp. 1-10.

Mackinnon, J.G. (1996). "Numerical distribution functions for unit root and cointegration tests", Journal of Applied Econometrics, 11 (6), pp. 601-618.

Maddala, G.S. y Kim, I. (2002). Unit roots, cointegration, and structural changes. Cambridge, Cambridge University Press. 
Mishkin, F.S. (1992). "Is the Fisher effect for real?: A reexamination of the relationship between inflation and interest rates", Journal of Monetary Economics, 30 (2), pp. 195-215.

Pesaran, M.H.; Smith, L.V. y Smith, R.P. (2007). "What if the UK or Sweden had joined the Euro in 1999? An empirical evaluation using a global VAR", International Journal of Finance \& Economics, 12 (1), pp. 55-87.

Philips, P. (1986). "Understanding spurious regression in econometrics", Journal of Econometrics, 33 (3), pp. 311-340.

Reade, J. y Volz, U. (2009a). “Leader of the Pack? German monetary dominance in Europe prior to EMU”, Economic Series Working Papers, Department of Economics, 419, University of Oxford.

Reade, J. y Volz, U. (2009b). "Too much to lose, or more to gain? Should Sweden join the euro?”, Discussion Paper Series, Department of Economics, 442, august.

Sander, H. y Kleimeier, S. (2006). "Convergence of interest rate pass-through in a wider Eurozone?", Economic Systems, 30 (4), pp. 405-423.

Sørensen, C. y Werner, T. (2006). "Bank interest rate pass-through in the euro area: A cross country comparison”, European Central Bank Working Paper, 580.

Weber, E. (2005). "British interest rate convergence between the US and Europe: A recursive cointegration analysis", The ICFAI Journal of Monetary Economics, 4 (4), pp. 29-47. 